\title{
Linear Dynamically Varying LQ Control of Nonlinear Systems over Compact Sets
}

\author{
Stephan Bohacek and Edmond A. Jonckheere
}

\begin{abstract}
Linear-quadratic controllers for tracking natural and composite trajectories of nonlinear dynamical systems evoluting over compact sets are developed. Typically, such systems exhibit "complicated dynamics," i.e., have nontrivial recurrence. The controllers, which use small perturbations of the nominal dynamics as input actuators, are based on modeling the tracking error as a linear dynamically varying (LDV) system. Necessary and sufficient conditions for the existence of such a controller are linked to the existence of a bounded solution to a functional algebraic Riccati equation (FARE). It is shown that, despite the lack of continuity of the asymptotic trajectory relative to initial conditions, the cost to stabilize about the trajectory, as given by the solution to the FARE, is continuous. An ergodic theory method for solving the FARE is presented. Furthermore, it is shown that wrapping the LDV controller around the nonlinear system secures a stable tracking dynamics. Finally, an example of controlling the Hénon map to follow an aperiodic orbit is presented.
\end{abstract}

Index Terms-Chaos, discrete-time Riccati equations, linear-quadratic control, nonlinear systems, time-varying systems, tracking, uncertain systems.

\section{INTRODUCTION}

A TYPICAL feature of nonlinear dynamical systems running over compact sets is that their phase portraits exhibit a variety of trajectories ranging from the trivial periodic orbits to the nonperiodic transitive orbits [18]. Sensitive dependence on initial conditions and other parameters [23] allows a preselected trajectory to be tracked-despite offset in initial conditions, extraneous disturbances and uncertainty on the dynamics - via a cheap control that acts as a small perturbation of the nominal dynamics. To formalize the above ideas, define the nominal and perturbed dynamics, respectively, as

$$
\begin{aligned}
& \theta(k+1)=f(\theta(k)), \quad \theta(0)=\theta_{0} \in \Theta, \\
& \varphi(k+1)=f(\varphi(k), u(k)), \quad \varphi(0)=\varphi_{0} \in \Theta .
\end{aligned}
$$

In the above, $\left\{\theta(k) \in \mathbb{R}^{n}: k \geq 0\right\}$ is the desired trajectory and $\varphi(k)$ is the state of the system under control $u(k) \in \mathbb{R}^{m}$, which is taken to be a small perturbation of the nominal dynamics, viz., $f(\varphi(k), 0)=f(\varphi(k))$. The dynamics are differentiable, viz., $f \in C^{1}\left(\mathbb{R}^{n} \times \mathbb{R}^{m}, \mathbb{R}^{n}\right)$ and the motion is restricted to a compact

Manuscript received August 1, 1997; revised October 29, 1998, November 16, 1999, and October 20, 2000. Recommended by Associate Editor A. Tesi. This work was supported by National Science Foundation under Grant ECS-9802594.

The authors are with the Department of Mathematics and Department of Electrical Engineering Systems, University of Southern California, Los Angeles, CA 90089-2563 (e-mail: bohacek@ math.usc.edu; jonckhee@eudoxus.usc.edu).

Publisher Item Identifier S 0018-9286(01)03603-0. $f$-invariant $(f(\Theta) \subseteq \Theta)$ subset of $\mathbb{R}^{n}$. The broad objective is to find a control $u$ such that $\lim _{k \rightarrow \infty}\|\varphi(k)-\theta(k)\|=0$.

This paper develops a controller of the form $u(k)=$ $F_{\theta(k)}(\varphi(k)-\theta(k))$, where the feedback $F_{\theta}$ is designed, for every $\theta \in \Theta$, from a linear approximation of $f(\varphi, u)$ around $(\theta, 0)$, and the gain $F_{\theta(k)}$ is "scheduled" so as to force $\varphi(k)$ to follow the desired trajectory $\theta(k)$. This is a specialized version of the LPV scheme [20], [3], where the parameter vector $\theta$, instead of being uncertain, is dynamically modeled. This justifies the terminology of linear dynamically varying ( $L D V)$ control.

An unusual feature of the LDV controller viewed as a tracking controller is that the gain is spatially varying and defined all over $\Theta$. As the first and most generic application, given an arbitrary desired trajectory $\{\theta(k): k=0, \ldots\}$, evaluating $F_{\theta}$ along the trajectory $\{\theta(k): k=0, \ldots\}$ yields the time-varying controller $F_{\theta(k)}$ that makes the nonlinear system $\varphi(k+1)=f\left(\varphi(k), F_{\theta(k)}(\varphi(k)-\theta(k))\right)$ asymptotically track $\theta(k+1)=f(\theta(k))$. More importantly, the globally defined controller $F_{\theta}$ becomes fully motivated in those specialized applications where there is a need to quickly adapt the tracking controller to a new reference trajectory without recomputing a new time-varying controller along the new trajectory. Specifically, having reached $\varphi\left(k_{0}\right) \approx \theta\left(k_{0}\right)$, one could track the trajectory $\left\{\tilde{\theta}(k): k \geq k_{0}\right\}$ starting at $\tilde{\theta}\left(k_{0}\right) \approx \theta\left(k_{0}\right)$ by switching from $\left\{F_{\theta(k)}: k=0, \ldots, k_{0}-1\right\}$ to $\left\{F_{\tilde{\theta}(k)}: k \geq k_{0}\right\}$. As shown in [6], switching among natural trajectories allows for such broader control objectives as targeting and periodic orbit avoidance. In orbital mechanics, switching among free orbits proved instrumental in NASA's experiment that involved steering a decommissioned satellite to a rendezvous with the Giaccobini-Zinner comet [11]. Along the related line of application of the X-33 program, there is a need to adapt the spacecraft controller to a change of launch-to-landing trajectory in case of failure and/or a change of landing site [15], [17].

The controller $F_{\theta}$ exists if and only if a solution $X_{\theta}$ to a functional algebraic Riccati equation (FARE) linking $X_{\theta}$ and $X_{f(\theta)}$ exists. The mathematical difficulty with this functional equation is to prove that the relevant solution is continuous, in which case $F_{\theta}$ is continuous.

Typically there is no closed form solution to the FARE. However, dynamical systems on compact sets, subject to some mild additional conditions, are known to have such ergodic properties as recurrence and transitivity [18], which can be put into use to construct an approximate solution of arbitrary accuracy.

The paper proceeds as follows. Section II formalizes the tracking control problem of interest and shows how the tracking 
error can be approximated as an LDV system. Section III formally develops LDV systems and the optimal linear-quadratic controllers for this class of systems. Section IV shows that these linear controllers are suitable for robustly stabilizing the nonlinear systems. Section V develops numerical techniques to compute the solution of the FARE. Section VI gives an example. Finally, the Appendix provides the proofs of the technical results.

\section{LINEAR DYNAMICALLY VARYING TRACKING ERROR DYNAMICS}

Define the tracking error

$$
x(k)=\varphi(k)-\theta(k) .
$$

Then, we obtain

$$
x(k+1)=f(\varphi(k), u(k))-f(\theta(k), 0) .
$$

The first degree Taylor approximation of $f(\varphi(k), u(k))$ around $\varphi(k)=\theta(k)$ and $u(k)=0$ yields

$$
x(k+1)=A_{\theta(k)} x(k)+B_{\theta(k)} u(k)+\eta(x(k), u(k), \theta(k))
$$

where

$$
A_{\theta}=\frac{\partial f}{\partial x}(\theta, 0), \quad B_{\theta}=\frac{\partial f}{\partial u}(\theta, 0)
$$

and $\eta(x(k), u(k), \theta(k))$ accounts for nonlinear terms, to be specific, since $f \in C^{1}$

$$
\begin{aligned}
\eta(x(k), u(k), \theta(k))= & \eta_{x}(x(k), u(k), \theta(k)) x(k) \\
& +\eta_{u}(x(k), u(k), \theta(k)) u(k)
\end{aligned}
$$

where

$$
\eta_{x_{i, j}}(x, u, \theta)=\int_{0}^{1}\left(\frac{\partial f_{i}}{\partial x_{j}}(t x+\theta, t u)-\frac{\partial f_{i}}{\partial x_{j}}(\theta, 0)\right) d t
$$

and

$$
\eta_{u_{i, j}}(x, u, \theta)=\int_{0}^{1}\left(\frac{\partial f_{i}}{\partial u_{j}}(t x+\theta, t u)-\frac{\partial f_{i}}{\partial u_{j}}(\theta, 0)\right) d t .
$$

Since $f \in C^{1}$ and $\Theta$ is compact, if $x$ and $u$ are bounded, then $\left(\partial f_{i} / \partial x_{j}\right)(t x+\theta, t u)-\left(\partial f_{i} / \partial x_{j}\right)(\theta, 0)$ is uniformly continuous in $x$ and $u$ for $t \in[0,1]$. In particular, for any $\varepsilon>0$, there exists a $\delta>0$ such that if $\|x\|,\|u\|<\delta$, then $\left|\left(\partial f_{i} / \partial x_{j}\right)(t x+\theta, t u)-\left(\partial f_{i} / \partial x_{j}\right)(\theta, 0)\right|<\varepsilon$ for $t \in[0,1]$. Therefore, $\left\|\eta_{x}(x, u, \theta)\right\|$ and $\left\|\eta_{u}(x, u, \theta)\right\|$ can be made as small as necessary by limiting the size of $u$ and $x$.

If $u$ and $x$ are small, the error dynamics can be approximated as

$$
x(k+1)=A_{\theta(k)} x(k)+B_{\theta(k)} u(k) .
$$

This is a linear system with coefficient matrices $A$ and $B$ that vary as $\theta(k)$ varies. Since $\theta(k)$ varies according to the dynamical equation (1), such an interconnection as (1) and (3) is called an LDV system.
LDV and linear parametrically varying (LPV) system can be unified under the so-called linear set-valued dynamically varying (LSVDV) systems characterized by a set-valued map $f$ [17]. LDV and LPV [5], [4] systems are the two extremes, the former characterized by the fact that $f(\theta)$ is reduced to a point, the latter characterized by $f(\theta)=\Theta$. Somewhere between the two extremes lies the case of slow systems characterized by $\left\|\theta^{\prime}-\theta\right\|<\Delta, \forall \theta^{\prime} \in f(\theta)$ [25], [3]. Under this condition, it is customary to postulate the existence of an analytic map $X: \Theta \rightarrow \mathbb{R}^{n \times n}$ such that $A_{\theta}^{\prime} X_{\theta+\delta} A_{\theta}-X_{\theta}<0$ for $\|\delta\|<\Delta$ from which stability follows. Here, instead of postulating analyticity or any other convenient property of some solution to an inequality, we prove continuity of the solution to a relevant equation [see (44)].

\section{LiNEAR DyNAMICALLY VARYING SySTEMS AND CONTROLS}

Before controllers for LDV systems can be developed, such systems must be formalized and the relevant stability and detectability concepts must be defined. For the purpose of control, an LDV system is defined as follows:

$$
\begin{aligned}
x(k+1) & =A_{\theta(k)} x(k)+B_{\theta(k)} u(k) \\
z(k) & =\left[\begin{array}{c}
C_{\theta(k)} x(k) \\
D_{\theta(k)} u(k)
\end{array}\right] \\
\theta(k+1) & =f(\theta(k))
\end{aligned}
$$

with

$$
\theta(0)=\theta_{o} \quad \text { and } \quad x(0)=x_{o}
$$

where
$f: \Theta \rightarrow \Theta$
$\Theta \subset \mathbb{R}^{n}$
$A: \Theta \rightarrow \mathbb{R}^{n \times n}$,
$B: \Theta \rightarrow \mathbb{R}^{n \times m}$,
$C: \Theta \rightarrow \mathbb{R}^{p_{1} \times n}$, and
$D: \Theta \rightarrow \mathbb{R}^{p_{2} \times m}$
$\theta(k) \in \Theta$
$x(k) \in \mathbb{R}^{n}$
$u(k) \in \mathbb{R}^{m}$
$z(k) \in \mathbb{R}^{p_{1}+p_{2}}$
continuous map; compact and $f$-invariant; functions that need not be continuous;

It is assumed that both states $x(k)$ and $\theta(k)$ are known at time point $k$.

It is often assumed that the system coefficient matrices $A, B$, $C$ and $D$ are continuous. We refer to such systems as continuous LDV systems. In Section II, it was assumed that $f \in C^{1}$ and $A$ and $B$ were defined to be the matrices of partial derivatives of $f$, so that $A$ and $B$ were continuous. Thus, the tracking error dynamics associated with system (1) and (2) can be approximated by a continuous LDV system. However, if a feedback $F: \Theta \rightarrow R^{m \times n}$ is used to stabilize a continuous LDV system, then the resulting closed-loop system is a continuous LDV system only if $F$ is continuous. Although this paper will focus on continuous LDV systems, we cannot a priori assume that the stabilizing feedback is continuous. Therefore, the definition of an LDV system should allow for discontinuous coeffi- 
cient matrices. Another motivation for allowing discontinuous LDV systems is to define a class large enough to encompass jump linear systems as discussed in [9].

From a mathematical perspective, a linear dynamically varying system is a family of linear time-varying systems indexed by the initial condition $\theta(0)$. If $\theta(0)$ is a fixed point, then the linear system with index $\theta(0)$ is time-invariant. If $\theta(0)$ is a periodic point, the linear system with index $\theta(0)$ is a periodically varying linear system. If $\theta(0)$ is an aperiodic point, the linear system is a linear time-varying system.

Since a linear dynamically varying system is an uncountable collection of linear time-varying systems, the concept of stability is slightly more complex in the dynamically varying case than it is in the time-varying case.

Definition 1: The linear dynamically varying system (8) is uniformly exponentially stable if for $u(k)=0$, there exist an $0<\alpha<1$ and a $\beta<\infty$ such that for all $\theta(0) \in \Theta$

$$
\|x(k)\| \leq \beta \alpha^{k}\|x(0)\| .
$$

System (8) is exponentially stable if for $u(k)=0$ and for each $\theta(0) \in \Theta$, there exist an $0<\alpha(\theta(0))<1$ and a $\beta(\theta(0))<\infty$ such that for all $x(j)$ and $j \leq k$

$$
\|x(k+j)\| \leq \beta(\theta(0)) \alpha(\theta(0))^{j}\|x(j)\| .
$$

System (8) is asymptotically stable if for $u(k)=0$, any $\|x(0)\|<\infty$ and any $\theta(0) \in \Theta$

$$
\|x(k)\| \rightarrow 0 .
$$

Thus, a linear dynamically varying system is exponentially stable if every linear system in the family of linear systems indexed by $\theta(0)$ is exponentially stable. The parameters, $\alpha(\theta)$ and $\beta(\theta)$, remain constant along a positive trajectory; i.e., $\alpha(f(\theta))=\alpha(\theta)$, but may vary discontinuously across different trajectories. Another difficulty with this stability concept is that it is possible that $\lim _{i} \alpha\left(\theta_{i}\right)=1$ while $\alpha\left(\lim _{i} \theta_{i}\right)<1$ for some sequence $\left\{\theta_{i} \in \Theta: i \geq 0\right\}$, in which case the system is exponentially stable, but not uniformly exponentially stable.

In the case of continuous LDV systems, asymptotic, exponential, and uniform exponential stability are equivalent.

Proposition 1: Assume that the function $A: \Theta \rightarrow \mathbb{R}^{n \times n}$ is continuous and $\Theta$ is compact. Then asymptotic, exponential and uniformly exponential stability are equivalent.

Proof: The proof is withheld until the Appendix.

Note that for general time-varying systems, exponential stability and asymptotic stability are not equivalent. However, in the case of continuous LDVs, continuity and compactness lead to the equivalence of these two forms of stability.

Since uniformly exponentially stable systems are inherently more robust than exponentially stable systems, it is preferable to remain within the confines of continuous LDV systems. Thus, when synthesizing a feedback for controlling a continuous LDV system, it is important to ensure that the feedback is not only asymptotically stabilizing, but also continuous. However, to maintain generality, an LDV system is considered stabilizable if there exists an exponentially stabilizing feedback, that is as follows.

Definition 2: System (8) is stabilizable if there exists a, not necessarily continuous, function $F: \mathbb{N} \times \Theta \rightarrow \mathbb{R}^{m \times n}$ such that for all $\theta(0) \in \Theta$ and for all $k,\|F(k, \theta(0))\| \leq \bar{F}(\theta(0))<\infty$ and the system

$$
\begin{array}{r}
x(k+1)=\left(A_{\theta(k)}+B_{\theta(k)} F(k, \theta(0))\right) x(k) \\
\theta(k)=f^{k}(\theta(0))
\end{array}
$$

is exponentially stable, that is, there exist $\alpha(\theta(0))$ and $\beta(\theta(0))$ such that

$$
\left\|\prod_{i=j}^{k-1}\left(A_{f^{i}(\theta(0))}+B_{f^{i}(\theta(0))} F(i, \theta(0))\right)\right\|^{\leq \beta(\theta(0)) \alpha^{k-j}(\theta(0))}
$$

where the factors of the matrix product are taken in the proper order.

Thus, the feedback that exists via the definition of stabilizability may not be uniformly bounded nor even continuous in $\theta(0)$. A feedback that is uniformly bounded and making the closed-loop system uniformly exponentially stable will be said to be uniformly stabilizing.

Along with stabilizability, a detectability concept is needed.

Definition 3: System (8) is uniformly detectable if there exists a, not necessarily continuous, function $L: \Theta \rightarrow \mathbb{R}^{n \times p}$ such that for $\theta \in \Theta,\left\|L_{\theta}\right\| \leq \bar{L}<\infty$ and the system

$$
\begin{aligned}
x(k+1) & =\left(A_{\theta(k)}+L_{\theta(k)} C_{\theta(k)}\right) x(k) \\
\theta(k) & =f^{k}(\theta(0))
\end{aligned}
$$

is uniformly exponentially stable. That is, there exist an $\alpha_{d}<1$ and a $\beta_{d}<\infty$ such that for all $\theta(0) \in \Theta, \| \prod_{i=j}^{k-1}\left(A_{f^{i}(\theta(0))}+\right.$ $\left.L_{f^{i}(\theta(0))} C_{f^{i}(\theta(0))}\right)\left\|\leq \beta_{d} \alpha_{d}^{k}\right\| x(0) \|$.

The conditions of stabilizability and uniform detectability, required to secure existence of an LDV controller, are slightly asymmetric. However, as can easily be shown by a duality argument, if the function $f$ is invertible, then uniform detectability can be weakened to detectability, which is exactly the dual of stabilizability.

Since stabilizability depends on $A, B$, and $f$, we will say that the triple $(A, B, f)$ is stabilizable to mean that system (8) is stabilizable. Similarly, we say that the triple $(A, C, f)$ is uniformly detectable to mean that system (8) is uniformly detectable.

Under the following assumptions, the existence of a uniformly stabilizing continuous linear dynamically varying quadratic controller is proved.

Assumption 1: The functions $A: \Theta \rightarrow \mathbb{R}^{n \times n}, C: \Theta \rightarrow$ $\mathbb{R}^{p \prime \times n}$ and $f: \Theta \rightarrow \Theta$ are such that $(A, C, f)$ is uniformly detectable.

Assumption 2: The functions $A: \Theta \rightarrow \mathbb{R}^{n \times n}, B: \Theta \rightarrow$ $\mathbb{R}^{n \times m}$ and $f: \Theta \rightarrow \Theta$ are such that $(A, B, f)$ is stabilizable.

Assumption 3: The functions $A, B, C, D$, and $f$ are continuous, $\Theta$ is compact and $D_{\theta}^{\prime} D_{\theta}>0$ for all $\theta \in \Theta$.

Our main result can now be formulated. 
Theorem 1: Suppose Assumptions 1, 2 and 3 hold. Then there exists a unique, bounded solution $X: \Theta \rightarrow \mathbb{R}^{n \times n}$ to the FARE

$$
\begin{aligned}
X_{\theta}= & A_{\theta}^{\prime} X_{f(\theta)} A_{\theta}-A_{\theta}^{\prime} X_{f(\theta)} B_{\theta}\left(D_{\theta}^{\prime} D_{\theta}+B_{\theta}^{\prime} X_{f(\theta)} B_{\theta}\right)^{-1} \\
& \times B_{\theta}^{\prime} X_{f(\theta)} A_{\theta}+C_{\theta}^{\prime} C_{\theta} .
\end{aligned}
$$

such that $X_{\theta}=X_{\theta}^{\prime} \geq 0$, and the feedback

$$
\begin{aligned}
u(k)=-\left(D_{\theta(k)}^{\prime} D_{\theta(k)}+B_{\theta(k)}^{\prime} X_{f(\theta(k))} B_{\theta(k)}\right)^{-1} & \\
& \times B_{\theta(k)}^{\prime} X_{f(\theta(k))} A_{\theta(k)} x(k)
\end{aligned}
$$

uniformly exponentially stabilizes system (8). For $\|x(0)\|<\infty$, this feedback minimizes

$$
\sum_{k=0}^{\infty}\|z(k)\|^{2}
$$

and $X$ is a uniformly continuous function.

Conversely, if Assumptions 1 and 3 hold and if there exists a bounded solution, $X$, to (9) such that $X_{\theta}=X_{\theta}^{\prime} \geq 0$, then system (8) is stabilizable and $X$ is continuous. In this case, if $X(k, N, \theta)$ solves the finite-horizon Riccati equation, i.e.,

$$
\begin{aligned}
X(k, N, \theta)= & A_{f^{k}(\theta)}^{\prime} X(k+1, N, \theta) A_{f^{k}(\theta)}+C_{f^{k}(\theta)}^{\prime} C_{f^{k}(\theta)} \\
& -A_{f^{k}(\theta)}^{\prime} X(k+1, N, \theta) B_{f^{k}(\theta)} \\
& \times\left(D_{f^{k}(\theta)}^{\prime} D_{f^{k}(\theta)}\right. \\
& \left.\quad+B_{f^{k}(\theta)}^{\prime} X(k+1, N, \theta) B_{f^{k}(\theta)}\right)^{-1} \\
& \times B_{f^{k}(\theta)}^{\prime} X(k+1, N, \theta) A_{f^{k}(\theta)}
\end{aligned}
$$

with

$$
X(N, N, \theta)=C_{f^{N}(\theta)}^{\prime} C_{f^{N}(\theta)},
$$

then $X(0, N, \theta) \rightarrow X_{\theta}$ uniformly in $\theta$.

Proof: The proof of this theorem is in the Appendix.

Remark 1: Stabilizability is a rather weak assumption. Indeed, stabilizability merely assumes that every trajectory is stabilizable. Given this obviously necessary condition, it is interesting to observe that continuity and compactness are all that are needed to prove the existence of a continuous and uniformly stabilizing controller.

Remark 2: The continuity of the cost $X$ is counterintuitive in the case where $f$ is sensitively dependent on initial conditions. It is easily seen that the general time-varying infinite-horizon optimal quadratic cost is continuous with respect to the uniform topology, that is, if $V_{0}$ is the optimal infinite-horizon quadratic cost associated with $\left\{A_{k}, B_{k}, C_{k}, D_{k}: k \geq 0\right\}$ and $\tilde{V}_{0}$ is the optimal cost associated with $\left\{\tilde{A}_{k}, \tilde{B}_{k}, \widetilde{C}_{k}, \widetilde{D}_{k}: k \geq 0\right\}$ and if

$$
\begin{gathered}
d\left(\left\{A_{k}, B_{k}, C_{k}, D_{k}: k \geq 0\right\},\left\{\tilde{A}_{k}, \tilde{B}_{k}, \tilde{C}_{k}, \tilde{D}_{k}: k \geq 0\right\}\right) \\
=\sup _{k}\left(\left\|A_{k}-\tilde{A}_{k}\right\|+\left\|B_{k}-\tilde{B}_{k}\right\|\right. \\
\left.\quad+\left\|C_{k}-\tilde{C}_{k}\right\|+\left\|D_{k}-\tilde{D}_{k}\right\|\right)
\end{gathered}
$$

is small, then $\left\|V_{0}-\tilde{V}_{0}\right\|$ is small. We say that $f$ has sensitive dependence on initial conditions if there exists an $r>0$ such that for all $\theta \in \Theta$ and $\varepsilon>0$, there exist a $\varphi \in \Theta,\|\varphi-\theta\|<\varepsilon$, and a $K<\infty$ such that $\left\|f^{K}(\theta)-f^{K}(\varphi)\right\|>r$. Hence, if $f$ has sensitivity to initial conditions, then

$$
\begin{gathered}
d\left(\left\{A_{f^{k}(\theta)}, B_{f^{k}(\theta)}, C_{f^{k}(\theta)}, D_{f^{k}(\theta)}: k \geq 0\right\},\right. \\
\left.\quad\left\{A_{f^{k}(\varphi)}, B_{f^{k}(\varphi)}, C_{f^{k}(\varphi)}, D_{f^{k}(\varphi)}: k \geq 0\right\}\right)
\end{gathered}
$$

may remain bounded from below for arbitrarily small $\|\theta-\varphi\|$. Thus, the time-varying system

$$
\left\{A_{f^{k}(\theta)}, B_{f^{k}(\theta)}, C_{f^{k}(\theta)}, D_{f^{k}(\theta)}: k \geq 0\right\}
$$

is discontinuous in $\theta$ and standard continuity results from timevarying control cannot be applied. Surprisingly, despite the fact that the distance between the time-varying systems indexed by $\theta$ and $\varphi$ remains bounded from below, Theorem 1 implies that $\left\|X_{\varphi}-X_{\theta}\right\|$ can be made small by taking $\|\varphi-\theta\|$ small enough. In particular, this continuity implies that the cost of stabilizing a periodic orbit is nearly the same as the cost to stabilize a nearby aperiodic one, whereas general time-varying results seem to imply that the cost of stabilizing these different orbits may be very different.

Remark 3: When evaluated along a particular trajectory, the FARE and hence the controller become time-invariant, periodically varying or time-varying depending on whether the trajectory is fixed, periodic or aperiodic, respectively.

\section{Stability AND Robustness OF NONLINEAR SySTEMS WITH LDV CONTROLLERS}

Here, we address the issue as to whether the LDV quadratic controller, guaranteed to stabilize the LDV system, also stabilizes the nonlinear system.

With the feedback given by Theorem 1 in place, the nonlinear system (3) becomes

$$
\begin{aligned}
x(k+1)= & \left(A_{\theta(k)}+B_{\theta(k)} F_{\theta(k)}\right) x(k) \\
& +\left(\eta_{x}(x(k), u(k), \theta(k))\right. \\
& \left.\quad+\eta_{u}(x(k), u(k), \theta(k)) F_{\theta(k)}\right) x(k) \\
\theta(k+1)= & f(\theta(k))
\end{aligned}
$$

where $\eta_{x}$ and $\eta_{u}$ are given by (5) and (6). By Theorem 1,

$$
\begin{aligned}
& x(k+1)=\left(A_{\theta(k)}+B_{\theta(k)} F_{\theta(k)}\right) x(k) \\
& \theta(k+1)=f(\theta(k))
\end{aligned}
$$

is uniformly exponentially stable. Thus, by [24, Th. 24.7], there exists an $\bar{\eta}>0$ such that if $\| \eta_{x}(x(k), u(k), \theta(k))+$ $\eta_{u}(x(k), u(k), \theta(k)) F_{\theta(k)} \| \leq \bar{\eta}$, then system (12) is uniformly exponentially stable. If we define

$$
\begin{aligned}
& \tilde{\eta}(x, u, \theta) \\
&:= \begin{cases}\eta_{x}(x, u, \theta)+\eta_{u}(x, u, \theta) F_{\theta}, & \text { if } \| \eta_{x}(x, u, \theta) \\
\bar{\eta} I, & +\eta_{u}(x, u, \theta) F_{\theta} \|<\bar{\eta}\end{cases} \\
& \text { otherwise }
\end{aligned}
$$


then

$$
\begin{aligned}
\tilde{x}(k+1)=( & \left(A_{\theta(k)}+B_{\theta(k)} F_{\theta(k)}\right. \\
& \left.+\tilde{\eta}\left(\tilde{x}(k), F_{\theta(k)} \tilde{x}(k), \theta(k)\right)\right) \tilde{x}(k)
\end{aligned}
$$

is uniformly exponentially stable. In this case, there exists a $G<$ $\infty$ such that

$$
\|\tilde{x}(k)\|<G\|\tilde{x}(0)\|
$$

for all $\theta(0) \in \Theta$. It was shown in Section II that $\eta_{x}(x(k), u(k), \theta(k)) \quad$ and $\quad \eta_{u}(x(k), u(k), \theta(k))$ can be made arbitrarily small by limiting the size of $x(k)$ and $u(k)$. Since $X_{\theta}$ is continuous, $F_{\theta}$ is continuous, and since $\Theta$ is compact, there exists a bound $\bar{F}<\infty$ such that $\left\|F_{\theta}\right\|<\bar{F}$. Thus, there exists an $\bar{x}>0$ such that if $\|x(k)\|<\bar{x}$ and $\|u(k)\|=\left\|F_{\theta(k)} x(k)\right\|<\bar{F} \bar{x}$, then

$$
\left\|\eta_{x}(x(k), u(k), \theta(k))+\eta_{u}(x(k), u(k), \theta(k)) F_{\theta(k)}\right\|<\bar{\eta} .
$$

Therefore, if

$$
\|\tilde{x}(0)\|<\frac{\bar{x}}{G}=: R_{\text {Capture }}
$$

then $\|\tilde{x}(k)\|<\bar{x}$ and $\left\|F_{\theta(k)} \tilde{x}(k)\right\|<\bar{F} \bar{x}$ and thus

$$
\begin{aligned}
& \| \eta_{x}\left(\tilde{x}(k), F_{\theta(k)} \tilde{x}(k), \theta(k)\right) \\
& \quad+\eta_{u}\left(\tilde{x}(k), F_{\theta(k)} \tilde{x}(k), \theta(k)\right) F_{\theta(k)} \|<\bar{\eta}
\end{aligned}
$$

and

$$
\tilde{\eta}\left(\tilde{x}(k), F_{\theta(k)} \tilde{x}(k), \theta(k)\right)=\eta\left(\tilde{x}(k), F_{\theta(k)} \tilde{x}(k), \theta(k)\right) .
$$

By uniqueness of the solution to a difference equation, we conclude that if $\tilde{x}(0)=x(0)$, with

$$
\|x(0)\|<R_{\text {Capture }}
$$

then $x(k)=\tilde{x}(k)$ and system (12) is uniformly exponentially stable.

Remark 4: For $x$ and $u$ bounded, the nonlinearity term $\eta$ in (12) is clearly a bounded feedback wrapped around the LDV plant, so that the natural way to reduce the effect of $\eta$, and amplify the domain of attraction, is an $H^{\infty}$ design. This approach is pursued in [6] and [7].

\section{EXPLOITING ERGODicity to SOLVE the FunCtional ALGEBRAIC RICCATI EQUATION (FARE)}

Many methods can be devised to solve the FARE. Here we investigate, in detail, a method based on the ergodic property of recurrence; other methods include the jump linear approximation [1], [9], [10], and the method based on iterating the Riccati recursion (11).

\section{A. Solving FARE Over Recurrent Set}

For notational convenience, define the Riccati map

$$
\begin{aligned}
\rho_{\theta}(X)= & A_{\theta}^{\prime} X A_{\theta}+C_{\theta}^{\prime} C_{\theta}-A_{\theta}^{\prime} X B_{\theta}\left(D_{\theta}^{\prime} D_{\theta}+B_{\theta}^{\prime} X B_{\theta}\right)^{-1} \\
& \times B_{\theta}^{\prime} X A_{\theta} .
\end{aligned}
$$

With this notation, the FARE can be written $X_{\theta}=\rho_{\theta}\left(X_{f(\theta)}\right)$.
By definition, the point $\theta$ is recurrent if for an arbitrary $\delta>$ 0 , there exists an $N(\delta)<\infty$ such that $\left\|\theta-f^{N(\delta)}(\theta)\right\|<$ $\delta$. Let $R(f)$ denote the set of recurrent points. Since $R(f)$ is invariant $(f(R(f)) \subseteq R(f))$, the FARE can be restricted to $R(f)$, and, furthermore, the solution $X_{\theta}$ can be extended by continuity from $R(f)$ to $\overline{R(f)}$. Recall that for a generic class of diffeomorphisms, $R(f)$ is the largest set where nontransient behavior occurs [18].

Take $\theta \in R(f)$. Since $X$ is continuous, for $\varepsilon>0$, there exists a $\delta>0$ such that $\left\|X_{\theta}-X_{f^{N(\delta)}(\theta)}\right\|<\varepsilon$. It follows that

$\left\|\rho_{\theta} \circ \rho_{f(\theta)} \circ \cdots \circ \rho_{f^{N(\delta)-1}(\theta)}\left(X_{f^{N(\delta)}(\theta)}\right)-X_{f^{N(\delta)}(\theta)}\right\|<\varepsilon$.

If we define

$$
\tilde{\rho}_{\theta}(Y)=\rho_{\theta}(Y)+X_{f^{N(\delta)}(\theta)}-X_{\theta}
$$

we have

$$
\tilde{\rho}_{\theta} \circ \rho_{f^{1}(\theta)} \circ \cdots \rho_{f^{N(\delta)-1}(\theta)}\left(X_{f^{N(\delta)}(\theta)}\right)=X_{f^{N(\delta)}(\theta)}
$$

and $\left\|\tilde{\rho}_{\theta}-\rho_{\theta}\right\|<\varepsilon$. Replacing $\tilde{\rho}_{\theta}$ with $\rho_{\theta}$ in the above yields the approximate equation for $X_{f^{N(\delta)}(\theta)}$

$$
\rho_{\theta} \circ \rho_{f^{1}(\theta)} \circ \cdots \rho_{f^{N(\delta)-1}(\theta)}(Y)=Y .
$$

It turns out that $Y, X_{f^{N(\delta)}(\theta)}$ and $X_{\theta}$ are "close."

Theorem 2: Assume that $f$ is a diffeomorphism and $R(f)$ is structurally stable [8]. Let $\theta$ be a recurrent point and let $\varepsilon>0$. Then there exists a $\delta>0$ such that $\left\|\theta-f^{N(\delta)}(\theta)\right\|<\delta$ implies that $\left\|X_{\theta}-Y\right\|<\varepsilon$ where $X$ solves the FARE and $Y$ solves (15).

Proof: The definition of the structural stability of $R(f)$ and the proof of this theorem are given in the Appendix.

Clearly, an approximate solution to the FARE is given by the fixed point of (15), which can be found as follows. Since $f$ is a diffeomorphism, $A_{\theta}$ is invertible for all $\theta \in \Theta$, and if we define

$$
\begin{aligned}
\alpha_{\theta} & =A_{\theta}^{-1} \\
\beta_{\theta} & =A_{\theta}^{-1} B_{\theta}\left(D_{\theta}^{\prime} D_{\theta}\right)^{-1} B_{\theta}^{\prime}, \\
\gamma_{\theta} & =C_{\theta}^{\prime} C_{\theta} A_{\theta}^{-1}, \\
\delta_{\theta} & =A_{\theta}^{\prime}+C_{\theta}^{\prime} C_{\theta} A_{\theta}^{-1} B_{\theta}\left(D_{\theta}^{\prime} D_{\theta}\right)^{-1} B_{\theta}^{\prime}
\end{aligned}
$$

then it can be shown [12] that

$$
\rho_{\theta}(Y)=\left(\gamma_{\theta}+\delta_{\theta} Y\right)\left(\alpha_{\theta}+\beta_{\theta} Y\right)^{-1}
$$

and

$$
\rho_{\theta} \circ \rho_{f^{1}(\theta)} \circ \cdots \rho_{f^{N-1}(\theta)}(Y)=(\tilde{\gamma}+\tilde{\delta} Y)(\tilde{\alpha}+\tilde{\beta} Y)^{-1}
$$

where

$$
\left[\begin{array}{cc}
\tilde{\alpha} & \tilde{\beta} \\
\tilde{\gamma} & \tilde{\delta}
\end{array}\right]=\left(\prod_{k=0}^{N-1}\left[\begin{array}{ll}
\alpha_{f^{k}(\theta)} & \beta_{f^{k}(\theta)} \\
\gamma_{f^{k}(\theta)} & \delta_{f^{k}(\theta)}
\end{array}\right]\right) .
$$

Equation (16) can be solved by finding a $Y$ such that

$$
\left(\prod_{k=0}^{N-1}\left[\begin{array}{ll}
\alpha_{f^{k}(\theta)} & \beta_{f^{k}(\theta)} \\
\gamma_{f^{k}(\theta)} & \delta_{f^{k}(\theta)}
\end{array}\right]\right)\left[\begin{array}{c}
I \\
Y
\end{array}\right] \subset\left\{\left[\begin{array}{c}
I \\
Y
\end{array}\right]\right\}
$$

where $\left\{\left[\begin{array}{l}I \\ Y\end{array}\right]\right\}$ is the span of $\left[\begin{array}{l}I \\ Y\end{array}\right]$. This invariant subspace problem can be solved by the usual methods. 

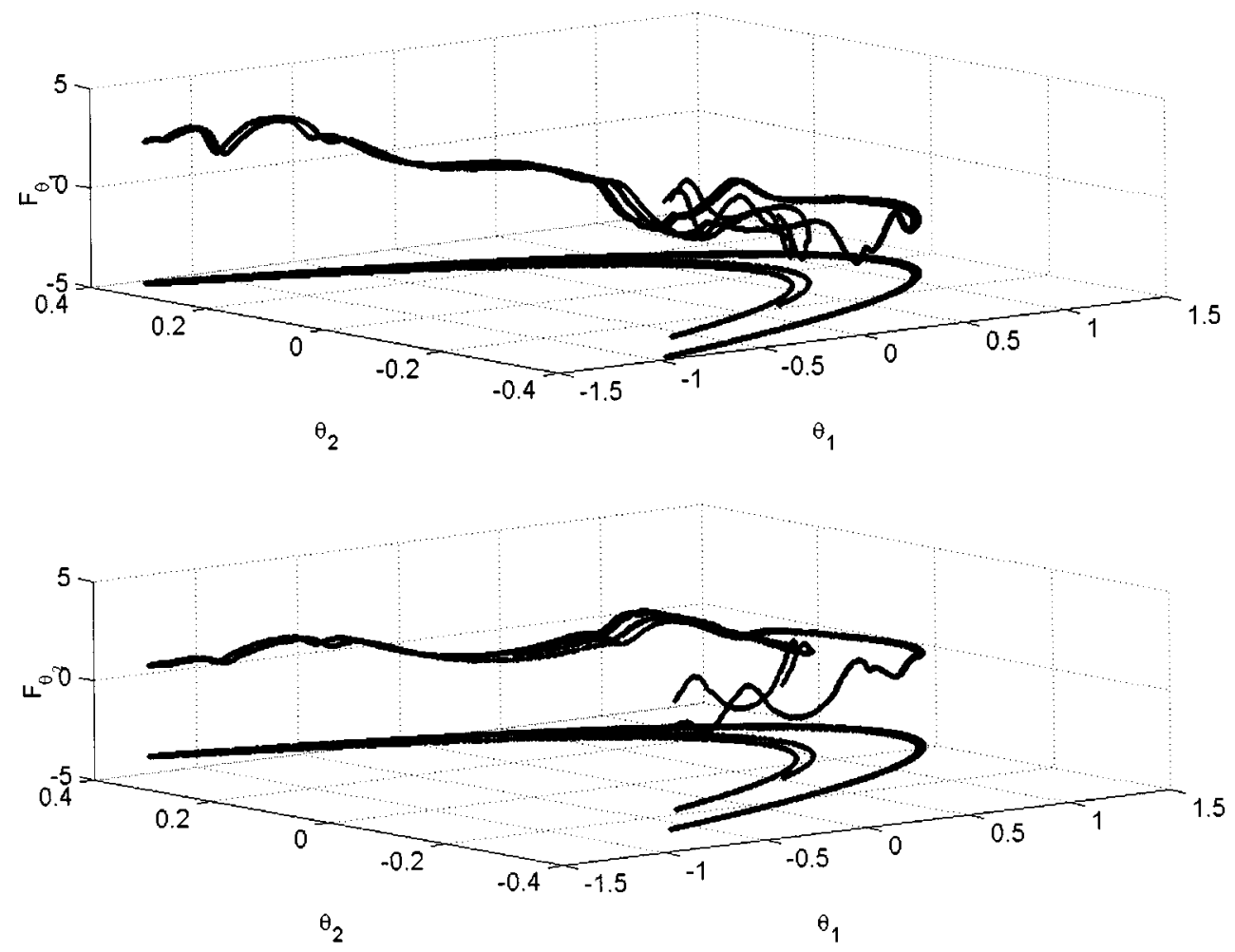

Fig. 1. Feedback $F_{\theta}=\left[\begin{array}{ll}F_{\theta_{1}} & F_{\theta_{2}}\end{array}\right]$ for the Hénon map. The plots show that the feedback is continuous in $\theta$. A plot of the attractor of the Hénon map in the $\left(\theta_{1}, \theta_{2},-6\right)$ plane is included for reference.

\section{EXAMPLE}

In the following, an LDV controller is devised for the Hénon system. The Hénon system is defined as

$$
\begin{aligned}
{\left[\begin{array}{c}
\gamma_{1}(k+1) \\
\gamma_{2}(k+1)
\end{array}\right] } & =\left[\begin{array}{l}
f_{1}(\gamma(k), u(k)) \\
f_{2}(\gamma(k), u(k))
\end{array}\right] \\
& =\left[\begin{array}{l}
1-(a+u(k)) \gamma_{1}(k)^{2}+\gamma_{2}(k) \\
b \gamma_{1}(k)
\end{array}\right]
\end{aligned}
$$

where $u$ is the control input. In this example, $a=1.4$ and $b=$ 0.3 . For these parameter values and $u \equiv 0$, it is known that the Hénon map has an attractor $\Theta$, that is, there exists an open set $V \supsetneq \Theta$ such that $\lim _{k \rightarrow \infty} d\left(f^{k}\left(\theta_{o}\right), \Theta\right)=0$ for all $\theta_{0} \in V$. This attractor is the crescent-shaped object shown in Fig. 1.

Define the associated LDV system by

$$
\begin{aligned}
& A_{\theta(k)}=\left.\frac{d f}{d \theta}\right|_{\theta(k), 0}=\left[\begin{array}{cc}
-2 a \theta_{1}(k) & 1 \\
b & 0
\end{array}\right] \\
& B_{\theta(k)}=\left.\frac{d f}{d u}\right|_{\theta(k), 0}=\left[\begin{array}{c}
-\theta_{1}^{2}(k) \\
0
\end{array}\right] .
\end{aligned}
$$

Numerical simulation [23] indicates that $\Theta$ is transitive, that is, for almost every $\theta_{o} \in \Theta$, the trajectory $\left\{f^{k}\left(\theta_{0}\right)\right\}$ enters every $\varepsilon$-neighborhood of every point $\theta \in \Theta$ for every $\varepsilon>0$.Therefore, iterating the time-varying Riccati equation (11) from a transitive point yields an approximate $X_{\theta}$. In this way, the gain $F: \Theta \rightarrow \mathbb{R}^{1 \times 2}$ is obtained and the closed-loop tracking error dynamics becomes

$$
\left[\begin{array}{c}
x_{1}(k+1) \\
x_{2}(k+1)
\end{array}\right]=\left(A_{\theta(k)}+B_{\theta(k)} F_{\theta(k)}\right)\left[\begin{array}{c}
x_{1}(k) \\
\left.x_{2}(k)\right)
\end{array}\right]+\left[\begin{array}{c}
\eta_{1}(k) \\
\eta_{2}(k)
\end{array}\right]
$$

where

$$
\begin{gathered}
\eta_{1}(k)=\left[\begin{array}{c}
\left.\left(-\varphi_{1}(k)-\theta_{1}(k)\right) F_{1_{\theta(k)}}-a\right) x_{1}(k) \\
-\left(\varphi_{1}(k)+x_{1_{\text {tra joct }}}(k)\right) F_{2_{\theta(k)}} x_{1}(k)
\end{array}\right]^{\prime}\left[\begin{array}{c}
\left.x_{1}(k)\right) \\
x_{2}(k)
\end{array}\right] \\
\eta_{2}(k)=0 \\
F_{\theta}=\left[\begin{array}{ll}
F_{1_{\theta}} & F_{2_{\theta}}
\end{array}\right] .
\end{gathered}
$$

Fig. 1 shows the feedback gain, $F$, for the LDV system (19). Note that the feedback is continuous on $\Theta$, the attractor of the Hénon map.

The objective in this example is to control the Hénon system so as to follow an aperiodic orbit described by $\theta(k+1)=$ $f(\theta(k), 0)$ with $\theta(0)=(0.6961,0.2088)$. Since the controlled system is only locally stable, control cannot begin until time $k=T$ when $\|\varphi(T)-\theta(T)\|<R_{\text {Capture }}$ and $R_{\text {Capture }}>0$ is the initial tracking error bound that ensures stability as defined in Section IV. Computer simulations indicate that the Hénon map is not distal and that $f \times f$ is ergodic [21]. Since $\{(\varphi, \theta) \in$ $\left.\Theta \times \Theta:\|\varphi-\theta\|<R_{\text {Capture }}\right\}$ has positive measure, the Poincaré recurrence theorem [18] on $\Theta \times \Theta$ implies that for almost every initial condition $\left(\theta_{o}, \varphi_{o}\right)$, we have $\|\varphi(T)-\theta(T)\|<R_{\text {Capture }}$ 


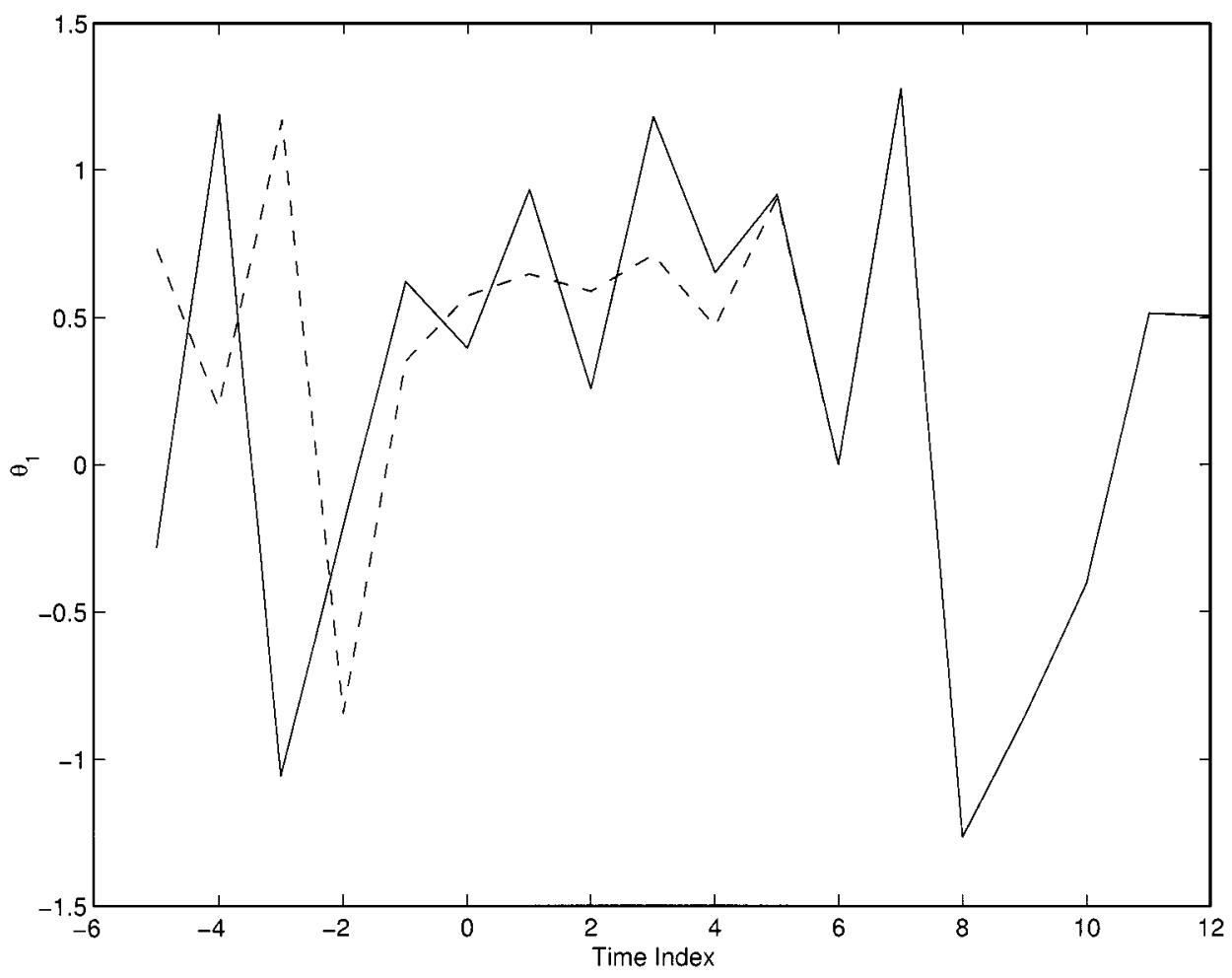

Fig. 2. Tracking: $\varphi_{1}$ (solid line) and $\theta_{1}$ (dotted line) are shown. At time $k=0,\|\varphi(0)-\theta(0)\|$ is small enough for the control to be safely turned on. Once the control is turned on, $\varphi$ tracks $\theta$.

for some $T$. For $k>T$, control force is applied via the control law $u(k)=F_{\theta(k)} x(k)$, where $F$ is given by the LDV quadratic control method of Section III.

Figs. 2 and 3 show the controlled trajectory, $\varphi_{1}(k)$, the desired trajectory, $\theta_{1}(k)$, and the tracking error, $x_{1}(k)=\varphi_{1}(k)-$ $\theta_{1}(k)$. At time index 0 , the error is small enough to safely turn the control on (i.e., $T=0$ ) and $\varphi_{1}$ tracks the desired trajectory. After the control is applied the error may increase beyond $R_{\text {Capture. }}$ Extensive simulation imply that if

$$
\begin{aligned}
& \sqrt{\left(\frac{1}{1.3}\right)^{2}\left(\varphi_{1}(T)-\theta_{1}(T)\right)^{2}+\left(\frac{1}{0.4}\right)^{2}\left(\varphi_{2}(T)-\theta_{2}(T)\right)^{2}} \\
& \quad<0.15
\end{aligned}
$$

the system remains stable, where the scaling factors account for the fact that $\theta_{1} \in[-1.3,1.3]$ and $\theta_{2} \in[-0.4,0.4]$.

An example of controlling the Hénon map to avoid its fixed point is available in [6].

\section{CONCLUSION}

LDV controllers for tracking natural and composite trajectories of nonlinear dynamical systems running over compact sets have been developed. The necessary and sufficient conditions for the existence of such controllers are rather weak and are equivalent to the existence of a bounded positive semidefinite solution to the FARE. If the dynamical system has adequate ergodic properties, there are many techniques for computing the solution to the FARE.
The LDV theory complements the popular LPV/gain scheduling theory by focusing on the extreme case of known parameter dynamics. Mathematically, the LMI of LPV design is pushed to the extreme situation of an equation linking the values of the solution for two successive values of the parameters, with the inescapable problem of proving continuity of the solution. Such equations, referred to as functional, are indeed notorious for generating badly behaved solutions, so that the LDV limit to the LPV theory was due to involve some mathematical difficulties.

It is hoped that the LSVDV theory, along with its continuous-time and $H^{\infty}$ counterparts [16], [6], [7] will emerge as a unification of the various gain scheduling concepts.

\section{APPENDIX}

Since a linear dynamically varying system is a collection of time-varying systems, the following time-varying Lyapunov stability theorem will prove useful.

Proposition 2: Assume $(A, C, f)$ is uniformly detectable. Then there exists an $\alpha\left(\theta_{0}\right) \in[0,1)$ and a $\beta\left(\theta_{0}\right)<\infty$ such that for $\theta(0)=\theta_{o}$ and any $x(k) \in \mathbb{R}^{n}$

$$
\|x(k+j)\| \leq \beta\left(\theta_{o}\right) \alpha\left(\theta_{o}\right)^{j}\|x(k)\|
$$

if and only if there exists a sequence $\left\{X_{f^{k}\left(\theta_{o}\right)}: k \geq 0\right\}$ with $\left\|X_{f^{k}\left(\theta_{o}\right)}\right\| \leq \bar{X}\left(\theta_{o}\right)<\infty$ and $X_{f^{k}\left(\theta_{o}\right)}=X_{f^{k}\left(\theta_{o}\right)}^{\prime} \geq 0$ such that

$$
A_{f^{k}\left(\theta_{o}\right)}^{\prime} X_{f^{k+1}\left(\theta_{o}\right)} A_{f^{k}\left(\theta_{o}\right)}-X_{f^{k}\left(\theta_{o}\right)} \leq-C_{f^{k}\left(\theta_{o}\right)}^{\prime} C_{f^{k}\left(\theta_{o}\right)}
$$




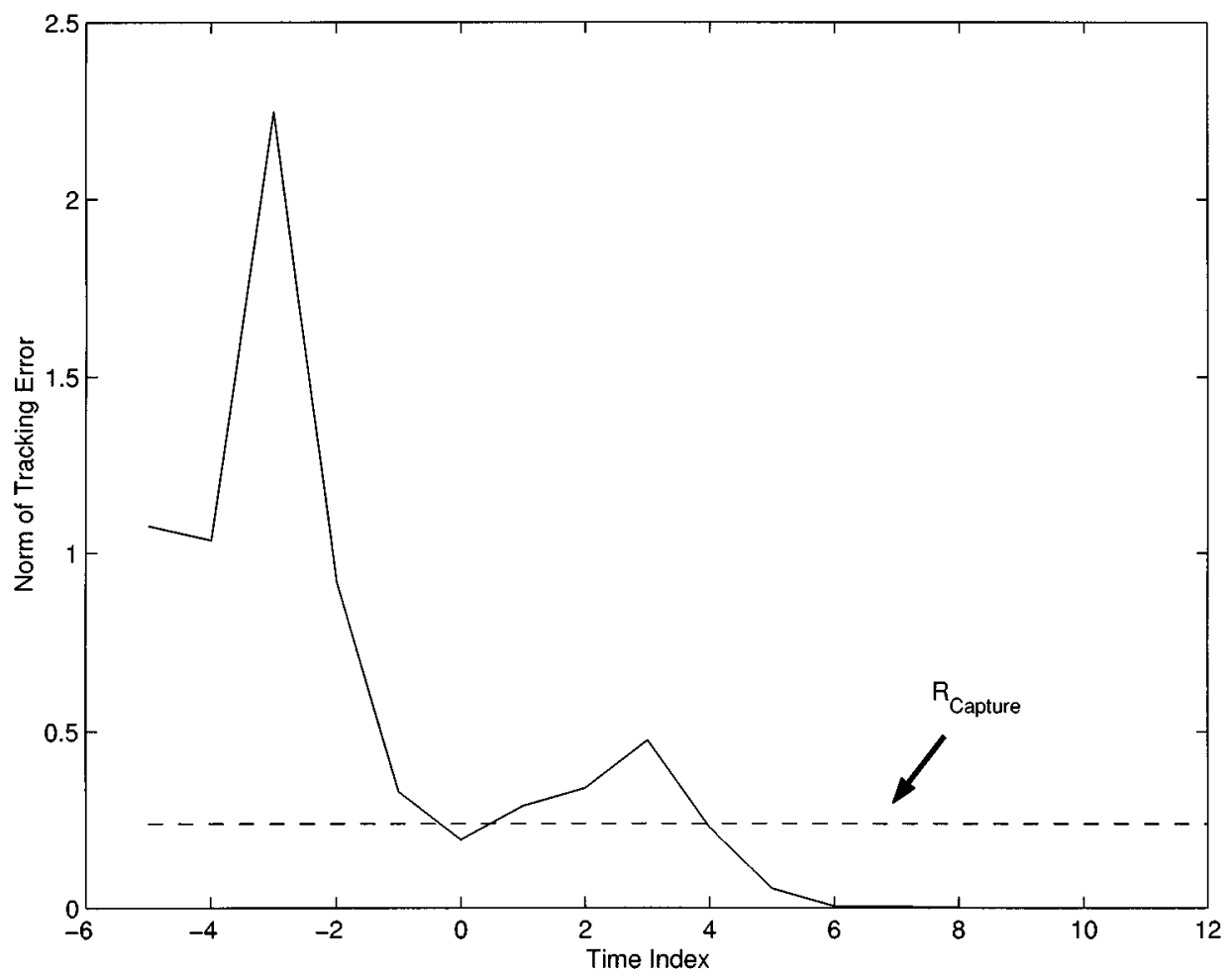

Fig. 3. Tracking error: At time $k=0$, the tracking error (solid line) is small enough, i.e., $\|\varphi(0)-\theta(0)\|<R_{\text {Capturc }}$, where $R_{\text {Capturc }}=0.15$. Hence, control is initiated at $k=0$. However, the tracking error exceeds $R_{\text {Capture }}$ for the next few time steps. This is acceptable, and the tracking error converges to zero.

Furthermore, if (22) is satisfied, then $\alpha(\theta)$ and $\beta(\theta)$ can be taken to only depend on the bound $\bar{X}\left(\theta_{o}\right)$ and on $\alpha_{d}$ and $\beta_{d}$ in the definition of uniform detectability.

Proof: For $\theta(0)$ fixed, the resulting LDV system is a linear time-varying system. Thus, the theorem is simply a statement regarding the stability of linear time-varying systems and can be found in [14].

Corollary 1: Assume $(A, C, f)$ is uniformly detectable. Then there exist an $\alpha<1$ and a $\beta<\infty$ such that

$$
\|x(k)\| \leq \beta \alpha^{k}\|x(0)\|
$$

if and only if there exists a uniformly bounded function $X: \Theta \rightarrow$ $\mathbb{R}^{n \times n}$ with $X_{\theta}=X_{\theta}^{\prime} \geq 0$ such that for all $\theta \in \Theta$

$$
A_{\theta}^{\prime} X_{f(\theta)} A_{\theta}-X_{\theta} \leq-C_{\theta}^{\prime} C_{\theta}
$$

Proof: Since $X_{\theta}$ is uniformly bounded and the system is uniformly detectable, Proposition 2 can be applied at each $\theta \in$ $\Theta$.

\section{A. Proof of Proposition 1}

Proof: Clearly, uniformly exponential stability implies exponential stability, which implies asymptotic stability. It remains to be shown that asymptotic stability implies uniformly exponential stability. Define $T(k, \theta(0))$ such that

$$
x(k)=A_{\theta(k-1)} A_{\theta(k-2)} \cdots A_{\theta(0)} x(0)=T(k, \theta(0)) x(0) .
$$

We claim that

$$
\gamma=\sup _{k} \sup _{\theta \in \Theta}\|T(k, \theta)\|<\infty \text {. }
$$

Suppose this is not true, i.e., $\gamma=\infty$. Define $T(N)=$ $\sup _{\theta \in \Theta}\|T(N, \theta)\|$. Then $T(N) \rightarrow \infty$ as $N \rightarrow \infty$ and there is a sequence $N_{n}$ such that $T\left(N_{n}\right)>T(M)$ for $M<N_{n}$ and $T\left(N_{n}\right)$ is monotone increasing to infinity. Since $\Theta$ is compact and $T\left(N_{n}, \theta\right)$ is continuous in $\theta$, there exists a $\theta_{n}$ such that $\left\|T\left(N_{n}, \theta_{n}\right)\right\|=T\left(N_{n}\right)$. Since $\Theta$ is compact, $\left\{\theta_{n}\right\}$ contains a convergent subsequence $\theta_{n_{m}} \rightarrow \theta_{o} \in \Theta$. Thus

$$
\begin{aligned}
\left\|T\left(N_{n_{m}}, \theta_{n_{m}}\right)\right\|= & T\left(N_{n_{m}}\right) \geq T(M) \\
= & \sup _{\theta \in \Theta}\|T(M, \theta)\| \geq\|T(M, \theta)\| \\
& \quad \text { for all } M \leq N_{n_{m}}, \theta \in \Theta .
\end{aligned}
$$

Since the system is asymptotically stable, if $\theta(0)=\theta_{o}$, then $\|x(k)\| \rightarrow 0$. Thus, there exists a $P=N_{k}<\infty$ such that $\left\|T\left(P, \theta_{0}\right)\right\|<1 / 4$. Since $P<\infty, T(P, \theta)$ is continuous in $\theta$. Thus, there is a $\delta>0$ such that $\left\|\theta_{o}-\theta\right\|<\delta$ implies that $\left\|T\left(P, \theta_{o}\right)-T(P, \theta)\right\|<1 / 4$. Since $\theta_{n_{m}} \rightarrow \theta_{o}$, there exists an $\bar{m}<\infty$ such that if $m \geq \bar{m}$ then $\left\|\theta_{o}-\theta_{n_{m}}\right\|<\delta$, $N_{n_{m}}>P$ and $\left\|T\left(P, \theta_{o}\right)-T\left(P, \theta_{n_{m}}\right)\right\|<1 / 4$. It follows that $\left\|T\left(P, \theta_{n_{m}}\right)\right\| \leq 1 / 2$. However, $T\left(N_{n_{m}}, \theta_{n_{m}}\right)=T\left(N_{n_{m}}-\right.$ $\left.P, f^{P}\left(\theta_{n_{m}}\right)\right) T\left(P, \theta_{n_{m}}\right)$ so that

$$
\left\|T\left(N_{n_{m}}, \theta_{n_{m}}\right)\right\| \leq\left\|T\left(N_{n_{m}}-P, f^{P}\left(\theta_{n_{m}}\right)\right)\right\|\left\|T\left(P, \theta_{n_{m}}\right)\right\|
$$

which implies that

$$
2\left\|T\left(N_{n_{m}}, \theta_{n_{m}}\right)\right\| \leq\left\|T\left(N_{n_{m}}-P, f^{P}\left(\theta_{n_{m}}\right)\right)\right\| .
$$

This contradicts equation (25). Thus, the claim (24) is true.

Since the system is asymptotically stable, for any $\theta_{0} \in \Theta$, if $\theta(0)=\theta_{o}$ then $\|x(k)\| \rightarrow 0$. Thus, there is an $N\left(\theta_{o}\right)<\infty$ such that if $N \geq N\left(\theta_{o}\right)$, then $\left\|T\left(N, \theta_{o}\right)\right\|<1 / 4 \gamma$, where $\gamma$ is given 
by equation (24). With $N\left(\theta_{0}\right)<\infty, T\left(N\left(\theta_{0}\right), \theta\right)$ is continuous in $\theta$. Thus, there exists a $\delta\left(\theta_{o}, N\left(\theta_{o}\right)\right)>0$ such that if $\left\|\theta_{o}-\theta\right\|$ $<\delta\left(\theta_{o}, N\left(\theta_{o}\right)\right)$, then $\left\|T\left(N\left(\theta_{o}\right), \theta_{o}\right)-T\left(N\left(\theta_{o}\right), \theta\right)\right\|<1 / 4 \gamma$. Therefore, $\left\|T\left(N\left(\theta_{0}\right), \theta\right)\right\|<1 / 2 \gamma$, which implies that for $N \geq$ $N\left(\theta_{0}\right)$

$$
\begin{aligned}
\|T(N, \theta)\| & \leq\left\|T\left(N-N\left(\theta_{o}\right), f^{N\left(\theta_{o}\right)}(\theta)\right)\right\|\left\|T\left(N\left(\theta_{o}\right), \theta\right)\right\| \\
& <\gamma \frac{1}{2 \gamma}=\frac{1}{2} .
\end{aligned}
$$

Since $\delta(\theta, N(\theta))>0$, the set $\{B(\theta, \delta(\theta, N(\theta))) \cap \Theta: \theta \in \Theta\}$ is an open covering of $\Theta$. Since $\Theta$ is compact, there exists a finite subcovering, i.e., $\{B(\theta, \delta(\theta, N(\theta))) \cap \Theta: \theta \in I\}$ is an open covering and $I$ is a finite set. Set $\bar{N}=\max _{\theta \in I} N(\theta)<\infty$. For all $\varphi \in \Theta$, there exists a $\theta \in I$ such that $\|\varphi-\theta\|<\delta(\theta, N(\theta))$. Thus, by (26)

$$
\|T(N, \varphi)\|<\frac{1}{2} \quad \text { for } N \geq \bar{N}
$$

and

$$
\|T(m N, \varphi)\|<\left(\frac{1}{2}\right)^{m} \quad \text { for } N \geq \bar{N} .
$$

Define $\alpha$ and $\beta$ by

$$
\begin{aligned}
& 0<\alpha=\left(\frac{1}{2}\right)^{1 / \bar{N}}<1, \\
& \beta=\frac{1}{\alpha^{\bar{N}}} \max _{k \leq \bar{N} \sup }\|T(k, \theta)\|<\infty .
\end{aligned}
$$

Let $k=m \bar{N}+l$ with $l<\bar{N}$; then

$$
\begin{aligned}
\|T(k, \theta)\| & \leq\|T(m \bar{N}, \theta)\|\left\|T\left(l, f^{m \bar{N}}(\theta)\right)\right\| \\
& \leq\left(\frac{1}{2}\right)^{m}\left\|T\left(l, f^{m \bar{N}}(\theta)\right)\right\| \\
& \leq\left(\frac{1}{2}\right)^{m \bar{N} / \bar{N}} \beta \alpha^{\bar{N}} \leq \alpha^{m \bar{N}} \beta \alpha^{l}=\beta \alpha^{k} .
\end{aligned}
$$

\section{B. Proof of Theorem 1}

The first, and most difficult, problem is to show that stabilizability implies that there exists a uniformly bounded $X \geq 0$ that solves the functional Riccati equation (9). The second problem is to show that uniform detectability and a uniformly bounded $X \geq 0$ that solves (9) imply that the optimal control (10) is uniformly stabilizing and $X$ is continuous.

To show that stabilizability implies existence of a solution to (9), the finite horizon time-varying linear-quadratic controller will be examined. By stabilizability, for each initial condition $\theta_{0}$, this controller will be shown to exist and to be bounded along the trajectory $\left\{f^{k}\left(\theta_{0}\right)\right\}$. This in turn will imply that the infinitehorizon time-varying Riccati equation is actually of the form (9). Finally, it will be shown that the solution to (9) is uniformly bounded.

To show that a solution of (9) implies uniform stabilizability, standard techniques will be employed to show that the LDV controller is uniformly stabilizing. Lemma 5 will show that the positive semi-definite solution to (9) is unique. Finally, Lemma 6 will show that the positive-semidefinite solution to (9) is continuous and the finite-horizon solution to the time-varying Riccati equation (27) converges uniformly to the positive semi-definite solution to (9).

Lemma 1: If assumptions 1 and 2 hold and $C$ and $D$ are bounded, then for each $\theta_{0} \in \Theta$ and $k<\infty$, there exists an optimal control $u(k)=F\left(k, \infty, \theta_{o}\right) x(k)$, where $F\left(k, \infty, \theta_{o}\right)$ is given by equations (31) and (32). Furthermore, this control is exponentially stabilizing and for each $\theta \in \Theta$, the cost of this feedback given by (31) is finite.

Proof: Define the finite-horizon cost-to-go

$$
\begin{aligned}
V\left(k, N, \theta_{o}, x_{o}, u\right)= & x^{\prime}(N) C_{f^{N}\left(\theta_{o}\right)}^{\prime} C_{f^{N}\left(\theta_{o}\right)} x(N) \\
& +\sum_{i=k}^{N-1} x^{\prime}(i) C_{f^{i}\left(\theta_{o}\right)}^{\prime} C_{f^{i}\left(\theta_{o}\right)} x(i) \\
& +u^{\prime}(i) D_{f^{i}\left(\theta_{o}\right)}^{\prime} D_{f^{i}\left(\theta_{o}\right)} u(i) .
\end{aligned}
$$

Dynamic programming arguments show that

$$
\begin{aligned}
\inf _{u} V\left(k, N, \theta_{o}, x_{o}, u\right) & =V\left(k, N, \theta_{o}, x_{o}, u_{N}\right) \\
& =x_{k}^{\prime} X\left(k, N, \theta_{o}\right) x_{k}
\end{aligned}
$$

where

$$
\begin{aligned}
X(k, & \left.N, \theta_{o}\right) \\
= & A_{\theta(k)}^{\prime} X\left(k+1, N, \theta_{o}\right) A_{\theta(k)} \\
& +C_{\theta(k)}^{\prime} C_{\theta(k)}-A_{\theta(k)}^{\prime} X\left(k+1, N, \theta_{o}\right) B_{\theta(k)} \\
& \times\left(D_{\theta(k)}^{\prime} D_{\theta(k)}+B_{\theta(k)}^{\prime} X\left(k+1, N, \theta_{o}\right) B_{\theta(k)}\right)^{-1} \\
& \times B_{\theta(k)}^{\prime} X\left(k+1, N, \theta_{o}\right) A_{\theta(k)}
\end{aligned}
$$

subject to the terminal condition

$$
X\left(N, N, \theta_{0}\right)=C_{\theta(N)}^{\prime} C_{\theta(N)} .
$$

By Assumption 2, system (8) is stabilizable. Thus, there exists a sequence of suboptimal feedback matrices $\left\{F_{\theta(k), \ldots \theta(1), \theta(0)}: k \geq 0\right\}$ such that, if

$$
\bar{u}(k)=F_{\theta(k), \ldots \theta(1), \theta(0)} x(k)
$$

then $\|x(k)\| \leq \beta(\theta(0))(\alpha(\theta(0)))^{k-j}\|x(j)\|$. The definition of stabilizability implies that $\|u(k)\| \leq \bar{F}(\theta(0))\|x(k)\|$, where $\bar{F}(\theta(0))<\infty$. Furthermore, $C_{\theta}$ and $D_{\theta}$ are continuous and, therefore, bounded as $\left\|C_{\theta}\right\| \leq \bar{C}<\infty,\left\|D_{\theta}\right\| \leq \bar{D}<\infty$. Thus, with the suboptimal feedback in place and $0 \leq k<N$, it is not hard to show that the cost can be bounded as

$$
\begin{aligned}
\bar{V}\left(x_{o}, \theta_{o}\right) & =\lim _{N \rightarrow \infty} V\left(0, N, \theta_{o}, x_{o}, \bar{u}\right) \\
& \left.\leq\left(\bar{C}^{2}+\bar{F}^{2}\left(\theta_{o}\right)\right) \bar{D}^{2}\right)\left\|x_{o}\right\|^{2} \frac{\beta^{2}\left(\theta_{o}\right)}{1-\alpha^{2}\left(\theta_{o}\right)}<\infty .
\end{aligned}
$$

Similarly, the cost-to-go can be bounded as

$$
\begin{aligned}
& V\left(k, N, \theta_{o}, x_{o}, u_{N}\right) \\
& \quad \leq \lim _{N \rightarrow \infty} V\left(k, N, \theta_{o}, x_{o}, \bar{u}\right) \\
& \left.\quad \leq\left(\bar{C}^{2}+\bar{F}^{2}\left(\theta_{o}\right)\right) \bar{D}^{2}\right)\|x(k)\|^{2} \frac{\beta^{2}\left(\theta_{o}\right)}{1-\alpha^{2}\left(\theta_{o}\right)}
\end{aligned}
$$

where $\bar{u}$ is given by (29). Clearly $X\left(k, N, \theta_{0}\right)$ is monotone in- 
creasing and bounded as $N \rightarrow \infty$, so

$$
X\left(k, \infty, \theta_{o}\right)=\lim _{N \rightarrow \infty} X\left(k, N, \theta_{o}\right)
$$

exists. Therefore, allowing $N \rightarrow \infty$ in (27) yields

$$
\begin{aligned}
X(k, & \left.\infty, \theta_{o}\right) \\
= & A_{\theta(k)}^{\prime} X\left(k+1, \infty, \theta_{o}\right) A_{\theta(k)} \\
& +C_{\theta(k)}^{\prime} C_{\theta(k)}-A_{\theta(k)}^{\prime} X\left(k+1, \infty, \theta_{o}\right) B_{\theta(k)} \\
& \times\left(D_{\theta(k)}^{\prime} D_{\theta(k)}+B_{\theta(k)}^{\prime} X\left(k+1, \infty, \theta_{o}\right) B_{\theta(k)}\right)^{-1} \\
& \times B_{\theta(k)}^{\prime} X\left(k+1, \infty, \theta_{o}\right) A_{\theta(k)}
\end{aligned}
$$

For $k<\infty$, define

$$
\begin{aligned}
F\left(k, \infty, \theta_{o}\right)= & \left(D_{\theta(k)}^{\prime} D_{\theta(k)}+B_{\theta(k)}^{\prime} X\left(k, \infty, \theta_{o}\right) B_{\theta(k)}\right)^{-1} \\
& \times B_{\theta(k)}^{\prime} X\left(k, \infty, \theta_{o}\right) A_{\theta(k)} .
\end{aligned}
$$

To prove that this feedback is exponentially stabilizing, observe that by standard manipulation we get

$$
\begin{aligned}
\left(A_{\theta(k)}\right. & \left.+B_{\theta(k)} F_{\theta(k)}\right)^{\prime} X\left(k+1, \infty, \theta_{o}\right)\left(A_{\theta(k)}+B_{\theta(k)} F_{\theta(k)}\right) \\
& -X\left(k, \infty, \theta_{o}\right) \\
= & -\left[\begin{array}{ll}
C_{\theta(k)}^{\prime} & F_{\theta(k)}^{\prime}\left(D_{\theta(k)}^{\prime} D_{\theta(k)}\right)^{1 / 2}
\end{array}\right] \\
\times & {\left[\begin{array}{l}
C_{\theta(k)} \\
\left(D_{\theta(k)}^{\prime} D_{\theta(k)}\right)^{1 / 2} F_{\theta(k)}
\end{array}\right] }
\end{aligned}
$$

Furthermore

$$
\left(A+B F,\left[\begin{array}{l}
C \\
(D D)^{1 / 2} F
\end{array}\right], f\right)
$$

is uniformly detectable, since

$$
A+B F+\left[\begin{array}{ll}
H & -B\left(D^{\prime} D\right)^{-1 / 2}
\end{array}\right]\left[\begin{array}{l}
C \\
(D D)^{1 / 2} F
\end{array}\right]=A+H C
$$

is uniformly exponentially stable for the output injection feedback $H$ given by the uniform detectability assumption. Since $X\left(k, \infty, \theta_{o}\right)$ is bounded, Theorem 2 applied to (33) implies that the closed-loop system is exponentially stable.

Remark 5: This lemma is nothing more than the infinite horizon, time-varying linear-quadratic control along a trajectory (for more details on time-varying optimal control see [2] or [13]). However, we have not shown that the closed loop system is uniformly exponentially stable.

The above lemma implies that for all $\theta \in \Theta$

$$
X_{\theta}=\lim _{N \rightarrow \infty} X(0, N, \theta)
$$

exists and is finite, although we have not yet proved that it is uniformly bounded. Therefore, $X$ is a function $X: \Theta \rightarrow \mathbb{R}^{n \times n}$. This function is a solution to a FARE.

Lemma 2: If Assumptions 1 and 2 hold, the function

$$
\begin{aligned}
X: \Theta & \longrightarrow \mathbb{R}^{n \times n} \\
\theta & \mapsto X_{\theta}
\end{aligned}
$$

satisfies

$$
\begin{aligned}
X_{\theta}= & A_{\theta}^{\prime} X_{f(\theta)} A_{\theta}-A_{\theta}^{\prime} X_{f(\theta)} B_{\theta}\left(R_{\theta}+B_{\theta}^{\prime} X_{f(\theta)} B_{\theta}\right)^{-1} \\
& \times B_{\theta}^{\prime} X_{f(\theta)} A_{\theta}+C_{\theta}^{\prime} C_{\theta}
\end{aligned}
$$

Proof: Direct manipulation of (31).

As we show below, there is a bound on $X(0, N, \theta)$ that does not depend on $\theta$ or $N$, that is, $X(0, N, \theta)$ is uniformly bounded. This feature of stabilizable continuous LDV systems is surprising. It means that, although the stabilizability assumption only implies that there exists a controller with possibly unbounded cost, the compactness and continuity assumptions of LDV systems imply that there exists a control such that the cost to stabilize any trajectory is uniformly bounded.

Lemma 3: If Assumptions 1, 2, and 3 hold, then $X(0, N, \theta)$ is uniformly bounded.

Proof: Suppose $X(0, N, \theta)$ is not uniformly bounded. Define $\bar{X}(N)=\sup _{\theta \in \Theta}\|X(0, N, \theta)\|$. Since $N<\infty$, $X(0, N, \theta)$ is continuous in $\theta$. Since $\Theta$ is compact, there exists a $\theta(N)$ such that $\|X(0, N, \theta(N))\|=\bar{X}(N)$. Since, by our supposition, $\lim _{N \rightarrow \infty} \bar{X}(N)=\infty$, the sequence $\theta(N)$ is such that $\|X(0, N, \theta(N))\| \rightarrow \infty$. Since $\Theta$ is compact, there exists a subsequence, $\theta\left(N_{n}\right)$, such that $\theta\left(N_{n}\right) \rightarrow \theta_{o} \in \Theta$. Define $\theta_{n}=\theta\left(N_{n}\right)$. Then $\left\|X\left(0, N_{n}, \theta_{n}\right)\right\|=\bar{X}\left(N_{n}\right)$; thus, for all $\theta \in \Theta,\left\|X\left(0, N_{n}, \theta_{n}\right)\right\| \geq\left\|X\left(0, N_{n}, \theta\right)\right\|$.

By Lemma 1, $\|X(0, \infty, \theta)\|<\infty$ for all $\theta \in \Theta$. In particular, $\left\|X\left(0, \infty, \theta_{0}\right)\right\|<\infty$. Furthermore, it is assumed that $\left\|X\left(0, N_{n}, \theta_{n}\right)\right\| \rightarrow \infty$ monotonically as $n \rightarrow \infty$. Thus, there exists an $\bar{n}_{1}<\infty$ such that, if $n_{1} \geq \bar{n}_{1}$ then

$$
4\left\|X\left(0, \infty, \theta_{o}\right)\right\| \leq\left\|X\left(0, N_{n_{1}}, \theta_{n_{1}}\right)\right\|<\infty .
$$

Lemma 1 implies that the closed-loop system is exponentially stable. Thus, with the feedback in place, there is a $P<\infty$ such that for $\theta(0)=\theta_{o}$, we have $\|x(P)\|<(1 / 4)\left\|x_{o}\right\|$. Define

$$
U_{\theta_{0}}(k, \theta)=\prod_{l=0}^{k-1}\left(A_{f^{l}(\theta)}+B_{f^{l}(\theta)} F_{f^{l}\left(\theta_{o}\right)}\right)
$$

where the factors of the product are taken in the correct order. Then $U_{\theta_{o}}(k, \theta)$ is the state transition matrix using the feedback $F_{f^{k}\left(\theta_{o}\right)}$. When $\theta(0) \neq \theta_{o}$ the feedback in not optimal. However, when $\theta(0)=\theta_{o}$, the feedback is optimal and

$$
\left\|U_{\theta_{o}}\left(P, \theta_{o}\right)\right\|<\frac{1}{4} .
$$

Since $P<\infty, U_{\theta_{o}}(P, \theta)$ is continuous in $\theta$. Since $\theta_{n} \rightarrow \theta_{o}$, there exists an $\bar{n}_{2}<\infty$ such that if $n_{2} \geq \bar{n}_{2}$, then

$$
\left\|U_{\theta_{o}}\left(P, \theta_{o}\right)-U_{\theta_{o}}\left(P, \theta_{n_{2}}\right)\right\|<\frac{1}{4}
$$

Thus

$$
\left\|U_{\theta_{o}}\left(P, \theta_{n_{2}}\right)\right\|<\frac{1}{2}
$$

Define

$$
\begin{aligned}
& W_{\theta_{o}}(0, N, \theta) \\
& =\sum_{k=0}^{N-1}\left(U_{\theta_{o}}^{\prime}(k, \theta) C_{f^{k}(\theta)}^{\prime} C_{f^{k}(\theta)} U_{\theta_{o}}(k, \theta)+U_{\theta_{o}}^{\prime}(k, \theta)\right. \\
& \left.\quad \times F_{f^{k}\left(\theta_{o}\right)}^{\prime} D_{f^{k}(\theta)}^{\prime} D_{f^{k}(\theta)} F_{f^{k}\left(\theta_{o}\right)} U_{\theta_{o}}(k, \theta)\right) .
\end{aligned}
$$


Thus $x_{o}^{\prime} W_{\theta_{0}}(0, N, \theta) x_{o}+x_{o}^{\prime} U_{\theta_{o}}^{\prime}(N, \theta) C_{f^{N}(\theta)}^{\prime} C_{f^{N}(\theta)} U_{\theta_{o}}$ $(N, \theta) x_{0}$ is the finite-horizon cost using the feedback matrix $F_{f^{k}\left(\theta_{o}\right)}$. This is the optimal cost when $\theta(0)=\theta_{o}$ and $N=\infty$, so that $x_{o}^{\prime} W_{\theta_{o}}\left(0, \infty, \theta_{o}\right) x_{o}=x_{o}^{\prime} X\left(0, \infty, \theta_{o}\right) x_{o}$. Since $P<\infty, W_{\theta_{o}}(0, P, \theta)$ is continuous in $\theta$. Thus, there exists an $\bar{n}_{3}<\infty$ such that $n_{3} \geq \bar{n}_{3}$ implies that

$$
\left\|W_{\theta_{o}}\left(0, P, \theta_{n_{3}}\right)-W_{\theta_{o}}\left(0, P, \theta_{o}\right)\right\| \leq\left\|X\left(0, \infty, \theta_{o}\right)\right\|
$$

which implies that

$$
\begin{aligned}
& \left\|W_{\theta_{o}}\left(0, P, \theta_{n_{3}}\right)\right\| \\
& \quad \leq\left\|W_{\theta_{o}}\left(0, P, \theta_{n_{3}}\right)-W_{\theta_{o}}\left(0, P, \theta_{o}\right)\right\|+\left\|W_{\theta_{o}}\left(0, P, \theta_{o}\right)\right\| \\
& \quad \leq\left\|X\left(0, \infty, \theta_{o}\right)\right\|+\left\|X\left(0, \infty, \theta_{o}\right)\right\|=2\left\|X\left(0, \infty, \theta_{o}\right)\right\| .
\end{aligned}
$$

Set $n=\max \left(\bar{n}_{1}, \bar{n}_{2}, \bar{n}_{3}, P\right)$ and let $x_{\circ}$ be such that $\left\|x_{o}\right\|=$ 1 and $\left\|X\left(0, N_{n}, \theta_{n}\right)\right\|=x_{o}^{\prime} X\left(0, N_{n}, \theta_{n}\right) x_{o}$. Equations (35) and (39) imply that

$$
\begin{aligned}
x_{o}^{\prime} W_{\theta_{o}}\left(0, P, \theta_{n}\right) x_{o} & \leq 2 x_{o}^{\prime} X\left(0, \infty, \theta_{0}\right) x_{o} \\
& \leq \frac{1}{2} x_{o}^{\prime} X\left(0, N_{n}, \theta_{n}\right) x_{o} .
\end{aligned}
$$

Thus

$$
\begin{aligned}
& x_{o}^{\prime} X\left(0, N_{n}, \theta_{n}\right) x_{o}-x_{o}^{\prime} W_{\theta_{o}}\left(0, P, \theta_{n}\right) x_{o} \\
& \quad \geq x_{o}^{\prime} X\left(0, N_{n}, \theta_{n}\right) x_{o}-\frac{1}{2} x_{o}^{\prime} X\left(0, N_{n}, \theta_{n}\right) x_{o} \\
& \quad=\frac{1}{2} x_{o}^{\prime} X\left(0, N_{n}, \theta_{n}\right) x_{o} .
\end{aligned}
$$

Now, if we do not use the optimal control for the first $P$ steps, our cost must be at least as high as the optimal cost, that is, [recalling $V\left(0, N_{n}, \theta_{n}, x_{o}, u\right)$ is the cost with initial conditions $\theta_{n}$ and $x_{o}$ using control $u$ for $N_{n}$ time steps]

$$
\begin{array}{rl}
\min _{u} & V\left(0, N_{n}, \theta_{n}, x_{o}, u\right) \\
\leq & x_{o}^{\prime} W_{\theta_{o}}\left(0, P, \theta_{n}\right) x_{o} \\
& \quad+\min _{u} V\left(0, N_{n}-P, f^{P}\left(\theta_{n}\right), U_{\theta_{o}}\left(P, \theta_{n}\right) x_{o}, u\right) \\
\text { or } \quad & \\
\min _{u} V\left(0, N_{n}, \theta_{n}, x_{o}, u\right) \\
=x_{o}^{\prime} X\left(0, N_{n}, \theta_{n}\right) x_{o} \\
\leq \leq & x_{o}^{\prime} W_{\theta_{o}}\left(0, P, \theta_{n}\right) x_{o} \\
\quad & +x_{o}^{\prime} U_{\theta_{o}}^{\prime}\left(P, \theta_{n}\right) X\left(0, N_{n}-P, f^{P}\left(\theta_{n}\right)\right) U_{\theta_{o}}\left(P, \theta_{n}\right) x_{o}
\end{array}
$$

or

$$
\begin{aligned}
& x_{o}^{\prime} X\left(0, N_{n}, \theta_{n}\right) x_{o}-x_{o}^{\prime} W_{\theta_{o}}\left(0, P, \theta_{n}\right) x_{o} \\
& \quad \leq x_{o}^{\prime} U_{\theta_{o}}^{\prime}\left(P, \theta_{n}\right) X\left(0, N_{n}-P, f^{P}\left(\theta_{n}\right)\right) U_{\theta_{o}}\left(P, \theta_{n}\right) x_{o} .
\end{aligned}
$$

Thus, combining (37), (40), and (41) yields

$$
\begin{aligned}
& \frac{1}{2} x_{o}^{\prime} X\left(0, N_{n}, \theta_{n}\right) x_{o} \\
& \quad \leq x_{o}^{\prime} X\left(0, N_{n}, \theta_{n}\right) x_{o}-x_{o}^{\prime} W_{\theta_{o}}\left(0, P, \theta_{n}\right) x_{o} \\
& \quad \leq x_{o}^{\prime} U_{\theta_{o}}^{\prime}\left(P, \theta_{n}\right) X\left(0, N_{n}-P, f^{P}\left(\theta_{n}\right)\right) U_{\theta_{o}}\left(P, \theta_{n}\right) x_{o} \\
& \quad<\frac{1}{4}\left\|X\left(0, N_{n}-P, f^{P}\left(\theta_{n}\right)\right)\right\| .
\end{aligned}
$$

Thus, the contracting hypothesis leads to

$$
2\left\|X\left(0, N_{n}, \theta_{n}\right)\right\|<\left\|X\left(0, N_{n}-P, f^{P}\left(\theta_{n}\right)\right)\right\| .
$$

On the other hand, we obviously have

$$
\left\|X\left(0, N_{n}, \theta_{n}\right)\right\| \geq \| X\left(0, N_{n}-P, f^{P}\left(\theta_{n}\right) \| .\right.
$$

Thus, there is a clear contradiction between the above two inequalities. Therefore, $X(0, N, \theta)$ is uniformly bounded.

Lemmas 1-3 show that stabilizability implies that there exists a uniformly bounded solution to the functional equation (34).

Now we show the converse. That is, if $X \geq 0$ solves (34) and is bounded, then the LDV system (8) is stabilizable. In fact, it is uniformly stabilizable and $X$ is continuous.

With the solution $X$ to (34), we construct the optimal feedback

$$
F_{\theta}=-\left(R_{\theta}+B_{\theta}^{\prime} X_{f(\theta)} B_{\theta}\right)^{-1} B_{\theta}^{\prime} X_{f(\theta)} A_{\theta}
$$

and we prove the following.

Lemma 4: Suppose Assumptions 1 and 3 hold, and $X: \Theta \rightarrow \mathbb{R}^{n \times n}$ is a uniformly bounded solution to the functional algebraic Riccati equation (34) such that $X_{\theta}=X_{\theta}^{\prime} \geq 0$. If $u(k)=F_{\theta(k)} x(k)$, where $F_{\theta}$ is given by (43), then the resulting closed-loop system is uniformly exponentially stable. Therefore, system (8) is stabilizable.

Proof: Standard manipulation shows that

$$
\begin{aligned}
& \left(A_{\theta}+B_{\theta} F_{\theta}\right)^{\prime} X_{f(\theta)}\left(A_{\theta}+B_{\theta} F_{\theta}\right)-X_{\theta} \\
& =-\left[\begin{array}{ll}
C_{\theta}^{\prime} & F_{\theta}^{\prime}\left(D_{\theta}^{\prime} D_{\theta}\right)^{1 / 2}
\end{array}\right]\left[\begin{array}{l}
C_{\theta} \\
\left(D_{\theta}^{\prime} D_{\theta}\right)^{1 / 2} F_{\theta}
\end{array}\right]
\end{aligned}
$$

where

$$
\left(A+B F,\left[\begin{array}{l}
C \\
\left(D^{\prime} D\right)^{1 / 2} F
\end{array}\right], f\right)
$$

is uniformly detectable. Since $X$ is uniformly bounded, Corollary 1 implies that the closed-loop system is uniformly exponentially stable.

Remark 6: If Assumptions 1, 2, and 3 hold, Lemmas 1-3 imply that there exists a solution $X$ to (34) such that $X_{\theta}^{\prime}=$ $X_{\theta} \geq 0$. Thus, Lemma 4 implies that the resulting closed-loop system is uniformly exponentially stable.

Lemma 5: Suppose Assumptions 1 and 3 hold. If a solution $X$ to equation (34) exists with $X_{\theta}=X_{\theta}^{\prime} \geq 0,\left\|X_{\theta}\right\|<\bar{X}<\infty$, then it is unique.

Proof: By standard results of linear-quadratic control of time-varying systems, the time-varying Riccati equation associated with the linear time-varying system

$$
\begin{aligned}
x(k+1) & =A_{f^{k}\left(\theta_{o}\right)} x(k)+B_{f^{k}\left(\theta_{o}\right)} u(k) \\
z(k) & =\left[\begin{array}{c}
C_{f^{k}\left(\theta_{o}\right)} x(k) \\
D_{f^{k}\left(\theta_{o}\right)} u(k)
\end{array}\right]
\end{aligned}
$$

is unique. Since the solution $X$ to the functional Riccati equation (34) coincides with the solution to the time-varying Riccati equation, $X$ must also be unique.

Lemma 6: Suppose Assumptions 1 and 3 hold and there exists a solution $X$ to (34) such that $X_{\theta}=X_{\theta}^{\prime} \geq 0$. Suppose 
that there exists an $\bar{X}<\infty$ such that $\|X(0, k, \theta)\| \leq \bar{X}$ for all $\theta \in \Theta$ and $k$. Then $X$ is uniformly continuous and $X(0, k, \theta) \rightarrow X_{\theta}$ uniformly.

Proof: Suppose $X$ is not continuous. Then there exists a $\theta_{o} \in \Theta$, an $x_{o}$ with $\left\|x_{o}\right\|=1$, an $\varepsilon>0$ and a sequence $\left\{\theta_{n}\right\}$ with $\theta_{n} \rightarrow \theta_{o}$, such that

$$
x_{o}^{\prime} X_{\theta_{o}} x_{o}>x_{o}^{\prime} X_{\theta_{n}} x_{o}+\varepsilon, \quad \text { for all } n
$$

or

$$
x_{o}^{\prime} X_{\theta_{o}} x_{o}<x_{o}^{\prime} X_{\theta_{n}} x_{o}-\varepsilon, \quad \text { for all } n .
$$

Suppose (46) is true. Lemma 4 implies that the closed-loop system is asymptotically stable. Thus, there exists an $N<\infty$ such that $\left\|U_{\theta_{o}}\left(N, \theta_{o}\right)\right\|<(1 / 2) \sqrt{\varepsilon / 4 \bar{X}}$, where $U$ is defined by (36). Since $N<\infty, U_{\theta_{o}}(N, \theta)$ is continuous in $\theta$. Thus, there is an $n_{1}<\infty$ such that if $n \geq n_{1}$, then $\left\|U_{\theta_{o}}\left(N, \theta_{n}\right)-U_{\theta_{o}}\left(N, \theta_{o}\right)\right\|<(1 / 2) \sqrt{\varepsilon / 4 \bar{X}}$; thus, $\left\|U_{\theta_{o}}\left(N, \theta_{n}\right)\right\|<\sqrt{\varepsilon / 4 \bar{X}}$. Likewise, $W_{\theta_{o}}(0, N, \theta)$ is continuous in $\theta$, where $W$ is defined by (38). Thus, there is an $n_{2}<\infty$ such that if $n \geq n_{2}$, then $\| W_{\theta_{o}}\left(0, N, \theta_{o}\right)$ $-W_{\theta_{o}}\left(0, N, \theta_{n}\right) \|<\varepsilon / 4$. Set $n \geq \max \left(n_{1}, n_{2}\right)$. If we use the feedback $u(k)=F_{f^{k}\left(\theta_{o}\right)} x(k)$ for $k \leq N$, and the optimal feedback for $k>N$, then the cost will be at least as large as the optimal cost. That is

$$
\begin{aligned}
x_{o}^{\prime} X_{\theta_{n}} x_{o} \leq & x_{o}^{\prime} W_{\theta_{o}}\left(0, N, \theta_{n}\right) x_{o} \\
& +x_{o}^{\prime} U_{\theta_{o}}^{\prime}\left(N, \theta_{n}\right) X_{f^{N}\left(\theta_{n}\right)} U\left(N, \theta_{n}\right) x_{o} \\
\leq & x_{o}^{\prime} W_{\theta_{o}}\left(0, N, \theta_{o}\right) x_{o}+\frac{\varepsilon}{4}+\frac{\varepsilon}{4} \\
\leq & x_{o}^{\prime} X_{\theta_{o}} x_{o}+\frac{\varepsilon}{2}<x_{o}^{\prime} X_{\theta_{n}} x_{o}-\frac{\varepsilon}{2}
\end{aligned}
$$

where the last inequality follows from (46). Thus, $x_{0}^{\prime} X_{\theta_{n}} x_{0}<$ $x_{o}^{\prime} X_{\theta_{n}} x_{o}-(\varepsilon / 2)$, which is impossible. Thus, (46) must not hold.

Similarly, suppose (45) holds. By Lemma 4, $\left\|U_{\theta}(k, \theta)\right\| \leq$ $\beta \alpha^{k}$ for some $\beta<\infty$ and $0<\alpha<1$. Thus, there exists an $\bar{N}<$ $\infty$ such that for all $\theta \in \Theta$ and $N \geq \bar{N},\left\|U_{\theta}(N, \theta)\right\| \leq \sqrt{\varepsilon / 2 \bar{X}}$. By Assumption 3, $A$ and $B$ are bounded from above and $R$ is bounded from below. Thus, since $\left\|X_{\theta}\right\| \leq \bar{X}$, there exists a $\bar{F}<\infty$ such that $\left\|F_{\theta}\right\| \leq \bar{F}$ for all $\theta \in \Theta$. Therefore, $F_{\theta_{n}}$ contains a convergent subsequence, $\left\{F_{\theta_{n_{m}}}\right\}$. Let $\tilde{F}_{1}=\lim _{m \rightarrow \infty}$ $F_{\theta_{n_{m}}}$. Similarly, there exists a sub-subsequence $\theta_{n_{m_{l}}} \rightarrow \theta_{o}$ such that $\tilde{F}_{2}=\lim _{l \rightarrow \infty} F_{f\left(\theta_{n_{m_{l}}}\right)}$ exists. In this fashion, define $\tilde{K}_{k}$ for $k \leq N$. Redefine $\left\{\theta_{n}\right\}$ to be the subsequence such that $\lim _{n \rightarrow \infty} \bar{F}_{f^{k}\left(\theta_{n}\right)}=\tilde{K}_{k}$ for $k \leq N$. Let $P(k)=\prod_{j=0}^{k-1}$ $\left(A_{f^{j}\left(\theta_{o}\right)}+B_{f^{j}\left(\theta_{o}\right)} \tilde{K}_{j}\right)$; then $U_{\theta_{n}}\left(k, \theta_{n}\right) \rightarrow P(k)$ for $k \leq N$. Since $\left\|U_{\theta_{n}}\left(N, \theta_{n}\right)\right\| \leq \sqrt{\varepsilon / 2 \bar{X}},\|P(N)\| \leq \sqrt{\varepsilon / 2 \bar{X}}$. Furthermore, since the feedback $\tilde{K}_{k}$ is not necessarily optimal

$$
\begin{gathered}
x_{o}^{\prime} X_{\theta_{o}} x_{o} \leq x_{o}^{\prime}\left(\sum_{k=0}^{N-1} P^{\prime}(k) C_{f^{k}\left(\theta_{o}\right)}^{\prime} C_{f^{k}\left(\theta_{o}\right)} P(k)\right. \\
\left.+P^{\prime}(k) \tilde{K}_{k}^{\prime} R_{f^{k}\left(\theta_{o}\right)} \tilde{K}_{k} P(k)\right) x_{o} \\
+x_{o}^{\prime} P^{\prime}(N) X_{f^{N}\left(\theta_{o}\right)} P(N) x_{o}
\end{gathered}
$$

$$
\begin{aligned}
& \leq \lim _{n \rightarrow \infty} x_{o}^{\prime} \sum_{k=0}^{N-1} \\
& \quad \times\left(U_{\theta_{n}}^{\prime}\left(k, \theta_{n}\right) C_{f^{k}\left(\theta_{o}\right)}^{\prime} C_{f^{k}\left(\theta_{o}\right)} U_{\theta_{n}}\left(k, \theta_{n}\right)\right. \\
& \quad+U_{\theta_{n}}^{\prime}\left(k, \theta_{n}\right) F_{f^{k}\left(\theta_{n}\right)}^{\prime} R_{f^{k}\left(\theta_{o}\right)} \\
& \left.\quad \times F_{f^{k}\left(\theta_{n}\right)} U_{\theta_{n}}\left(k, \theta_{n}\right)\right) x_{o}+\frac{\varepsilon}{2} \\
& \leq \limsup _{n \rightarrow \infty} x_{o}^{\prime} X_{\theta_{n}} x_{o}+\frac{\varepsilon}{2}<x_{o}^{\prime} X_{\theta_{o}} x_{o}-\frac{\varepsilon}{2}
\end{aligned}
$$

where the last inequality follows from (45). Thus, (45) must be false. Therefore, $X$ is continuous. Since $\Theta$ is compact, $X$ is uniformly continuous.

Since $X$ is continuous, $X(0, N, \theta)$ is continuous, $X(0, N, \theta) \rightarrow X_{\theta}$ monotonically and $\Theta$ is compact, it follows that $X(0, N, \theta) \rightarrow X_{\theta}$ uniformly. For details, see [19].

\section{Proof of Theorem 2}

We prove that for $\varepsilon>0$, there exists a $\delta>0$ such that if $\left\|f^{N}(\theta)-\theta\right\|<\delta$ for some $N$, then $\left\|X_{\theta}-Y\right\|<\varepsilon$, where $X$ solves the FARE and $Y$ solves (15). Since $\theta$ is recurrent, given $\delta>0$, there exists an $N<\infty$ such that $\left\|f^{N}(\theta)-\theta\right\|<\delta$. It is assumed that $f$ is a diffeomorphism and that $\overline{R(f)}$ is structurally stable, i.e., for $\nu>0$ there exists a $\delta>0$ such that if $d_{C^{0}}(f, \tilde{f})<\delta$ then $d_{H}(R(f), R(\tilde{f}))<\nu$, where $d_{H^{\prime}}(\cdot, \cdot)$ is the Hausdorff metric and $d_{C^{0}}(f, \tilde{f})=\sup _{\theta}\|f(\theta)-\tilde{f}(\theta)\|$. Note that if $\overline{R(f)}$ is attracting, then structural stability of $\overline{R(f)}$ is a generic property [22]. Furthermore, the closure of the recurrent set of a hyperbolic system is structurally stable [18].

Lemma 7: Let $f$ be a diffeomorphism and $\theta$ be a recurrent point. For any $\delta>0$ there exist an $N<\infty$ and a continuous $\tilde{f}$ such that $\tilde{f}^{N}(\theta)=\theta$ and $d_{C^{0}}(f, \tilde{f})<\delta$.

Proof: Let $K$ be a connected compact set with $\Theta \subsetneq K$. $f \in C^{0}$ implies that there exists a $\gamma$ such that $\delta>\gamma>0$, and for $a, b \in K$

$$
\|a-b\|<\gamma \text { implies that }\|f(a)-f(b)\|<\delta .
$$

Similarly, $f^{-1} \in C^{0}$ implies that there exists a $\nu$ with $\delta>\nu>$ 0 such that for $\theta, \varphi \in K$

$$
\|\theta-\varphi\|<\nu \text { implies that }\left\|f^{-1}(\theta)-f^{-1}(\varphi)\right\|<\frac{\gamma}{3} .
$$

Now, since $\theta$ is recurrent, there exists an $N<\infty$ such that $\left\|f^{N}(\theta)-\theta\right\|<\nu$. Thus relation (48) implies that $\left\|f^{-1}(\theta)-f^{N-1}(\theta)\right\|<\gamma / 3$. Let $M$ be a smooth curve connecting $f^{-1}(\theta)$ and $f^{N-1}(\theta)$ such that if $a, b \in M$, then $\|a-b\|<\gamma(2 / 3)$ and $\min _{k=0, \ldots N-2} d\left(f^{k}(\theta), M\right)>\gamma_{2}>0$. Let $\gamma_{3}=\min \left((\gamma / 3)(1 / 2),\left(\gamma_{2} / 2\right)\right)$. The $C^{0}$ closing lemma [22] implies that there exists a $\tilde{f} \in C^{0}$ such that

1) $\tilde{f}(\varphi)=f(\varphi)$ for $\varphi \notin B\left(M, \gamma_{3}\right)$ where $B\left(M, \gamma_{3}\right)$ is the open $\gamma_{3}$-ball around $M$;

2) $\tilde{f}\left(f^{N-1}(\theta)\right)=\theta$; thus, $\tilde{f}^{N}(\theta)=\theta$;

3) $d_{C^{0}}(\tilde{f}, f)<\max \left(\operatorname{diam}\left(B\left(M, \gamma_{3}\right)\right)\right.$, $\left.\operatorname{diam}\left(f\left(B\left(M, \gamma_{3}\right)\right)\right)\right)$.

Since $\operatorname{diam}(M)<\gamma(2 / 3)$, and $\gamma_{3} \leq(\gamma / 3)(1 / 2)$, we have $\operatorname{diam}\left(B\left(M, \gamma_{3}\right)\right)<\gamma<\delta$. Since $\operatorname{diam}\left(B\left(M, \gamma_{3}\right)\right)<\gamma$, rela- 
tion (47) implies that $\operatorname{diam}\left(f\left(B\left(M, \gamma_{3}\right)\right)\right)<\delta$. It follows that $d_{C^{0}}(\tilde{f}, f)<\delta$ as desired.

Proposition 3: Let $f \in C^{1}$. Assume that the LDV system induced by $f$ is stabilizable, $(A, C, f)$ is uniformly detectable, $D_{\theta}^{\prime} D_{\theta}>0$ for all $\theta \in \Theta$, and $C$ and $D$ are continuous. Then, for all $\varepsilon>0$, there exists a $\delta>0$ such that if

$$
d_{C^{0}}(f, \tilde{f})+d_{H}(\Theta, \tilde{\Theta})<\delta
$$

then

$$
\left\|X_{\theta}-\tilde{X}_{\arg \min \{\|\tilde{\theta}-\theta\|: \tilde{\theta} \in \tilde{\Theta}\}}\right\|<\varepsilon, \quad \text { for all } \theta \in \Theta
$$

and

$$
\left\|\tilde{X}_{\tilde{\theta}}-X_{\arg \min \{\|\tilde{\theta}-\theta\|: \theta \in \Theta\}}\right\|<\varepsilon, \quad \text { for all } \tilde{\theta} \in \tilde{\Theta}
$$

where

$X$ positive-semidefinite solution to the FARE induced by $(A, B, C, D, f)$;

$\tilde{X} \quad$ solution induced by $(A, B, C, D, \tilde{f})$;

$\tilde{\Theta} \quad \tilde{f}$-invariant set.

Proof: See [8].

The above proposition implies that if $\varepsilon>0, \overline{R(f)}=\Theta$ and $\overline{R(f)}$ is structurally stable, there exists a $\delta>0$ such that if $d_{C^{0}}(f, \tilde{f})<\delta$ then $\left\|X_{\theta}-\tilde{X}_{\arg \min \{\|\tilde{\theta}-\theta\|: \tilde{\theta} \in \tilde{\Theta}\}}\right\|<\varepsilon$. Setting $\theta \in R(f)$, Lemma 7 provides an $N<\infty$ such that there exists an $\tilde{f}$ such that $d_{C^{0}}(f, \tilde{f})<\delta$ with $\tilde{f}^{N}(\theta)=\theta$. Therefore, $\tilde{X}_{\theta}=\rho_{\theta} \circ \rho_{\tilde{f}^{1}(\theta)} \circ \cdots \rho_{\tilde{f}^{N-1}}\left(\tilde{X}_{\theta}\right)$ where $\tilde{X}$ solves the FARE associated with the LDV system $(A, B, C, D, \tilde{f})$. Since $\tilde{f}^{k}(\theta)=f^{k}(\theta)$ for $k \leq N-1$, we have $\tilde{X}_{\theta}=\rho_{\theta} \circ \rho_{f^{1}(\theta)}$ $\circ \cdots \rho_{f^{N-1}(\theta)}\left(\tilde{X}_{\theta}\right)$, that is, $\tilde{X}_{\theta}$ solves (15) and $\left\|X_{\theta}-\tilde{X}_{\theta}\right\|<$ $\varepsilon$.

Remark 7: In numerical analysis language, Lemma 7 is the numerical stability of the algorithm-the computed fixed point $\theta$ is the exact fixed point for a nearby function. Proposition 3 is the numerical conditioning of the algorithm-a small perturbation of the function $f$ results in a small perturbation of the Riccati solution.

\section{REFERENCES}

[1] H. Abou-Kandil, G. Freiling, and G. Jank, "Solution and asymptotic behavior of coupled Riccati equations in jump linear systems," IEEE Trans. Automat. Contr., vol. 39, pp. 1631-1636, 1994.

[2] B. Anderson and J. Moore, "Detectability and stabilizability of timevarying discrete-time linear systems," SIAM J. Control Optim., vol. 19, no. 1 , pp. 20-32, 1981

[3] P. Apkarian, "Advanced gain-scheduling techniques for uncertain systems," IEEE Trans. Control Syst. Technol., vol. 6, pp. 21-32, Jan. 1998.

[4] P. Apkarian, P. Gahinet, and G. Becker, "Self-scheduled $H_{\infty}$ control of linear parameter-varying systems: Design example," Automatica, vol. 31, no. 9, pp. 1251-1261, 1995.

[5] G. Becker and A. Packard, "Robust performance of linear parameterically varying systems using parametrically-dependent linear feedback," Syst. Control Lett., vol. 23, pp. 205-215, 1995.

[6] S. Bohacek and E. Jonckheere, "Linear dynamically varying $H^{\infty}$ control of chaos," in Nonlinear Control Systems Design Symp. (NOLCOS), 1998, pp. 744-749.

[7] - "Linear dynamically varying $H^{\infty}$ control of systems with complicated asymptotic dynamics," SIAM J. Control Optim., 2001, to be published.

[8] - "Structural stability of LDV controllers," in Conf. Decision Control, Tampa, FL, Dec. 16-18, 1998, pp. 4630-4635.

[9] - "Linear dynamically varying (LDV) systems versus jump linear systems," in Amer. Control Conf., San Diego, CA, June 2-4, 1999, pp. 4024-4028.
[10] I. Borno and Z. Gajic, "Parallel algorithm for solving coupled algebraic Lyapunov equations of discrete-time jump linear systems," Comp. Math. Appl., vol. 30, pp. 1-4, 1995.

[11] W. Bown, "Mathematicians learn how to tame chaos," New Scientist, vol. 134 , no. 1823 , pp. 16-16, 1992.

[12] R. Bucy and L. Campbell, "Determination of steady state behavior for periodic discrete filtering problem," Comp. Math. Appl., vol. 15, no. 2, pp. 131-140, 1988

[13] R. Bucy and P. Joseph, Filtering for Stochastic Processes with Applications to Guidance. New York: Chelsea, 1987.

[14] A. Halanay and V. Ionescu, Time-Varying Discrete Linear Systems. Cambridge, MA: Birkhauser, 1994.

[15] J. Burken, private communication, 1999.

[16] E. Jonckheere and S. Bohacek, "Global topological aspects of continuous-time linear dynamically varying (LDV) control," IEEE Conf. Decision Control, pp. 2997-3002, Dec. 16-18, 1999.

[17] E. Jonckheere, P. Lohsoonthorn, and S. Bohacek, "From Sioux-City to the X-33," in Annual Reviews in Control. New York: Elsevier/Pergamon, 1999, vol. 23, pp. 91-108.

[18] A. Katok and B. Hasselblatt, Introduction to the Modern Theory of Dynamical Systems. Cambridge, U.K.: Cambridge Univ. Press, 1995.

[19] J. R. Munkres, Topology. Upper Saddle River, NJ: Prentice-Hall, 1999.

[20] A. Packard and M. Kantner, "Gain scheduling the LPV way," in Conf. Decision Contr., 1996.

[21] K. Petersen, Ergodic Theory. Cambridge, U.K.: Cambridge Univ. Press, 1983.

[22] S. Y. Pilyugin, The Space of Dynamical Systems with the $C^{0}$-Topology. New York: Springer-Verlag, 1991.

[23] C. Robinson, Dynamical Systems. Boca Raton, FL: CRC Press, 1995.

[24] W. Rugh, Linear System Theory. New Jersey: Prentice-Hall, 1996.

[25] F. Wu, X. H. Yang, A. Packard, and G. Becker, "Induced 1-2 norm control for LPV systems with bounded parameter variation rates," Int. J. Robust Nonlinear Control, vol. 6, pp. 983-998, 1996.

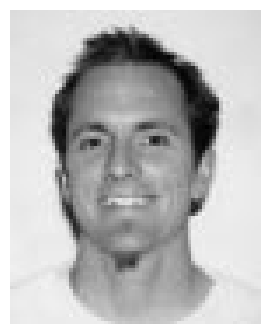

Stephan Bohacek received the B.S. degree in electrical engineering from the University of California at Berkeley, and the Ph.D. degree in electrical engineering from the University of Southern California, Los Angeles, in 1989 and 1999, respectively.

From 1989 to 1996, he worked in the area of audio signal processing for Wadia Digital and Theta Digital. Since 1999, he has been an Assistant Professor of Mathematics at the University of Southern California. His current areas of research include control theory, congestion control in computer networks, security in computer networks, and modeling neuromuscular-skeletal systems in mammals.

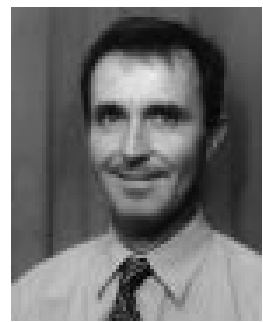

Edmond A. Jonckheere was born in Belgium in 1950. He received the M.S. degree in electrical engineering from the University of Louvain, Belgium, the Dr. Eng. degree in aerospace engineering from the University of Toulouse, France, and the Ph.D. degree in electrical engineering from the University of Southern California, Los Angeles, in 1973, 1975, and 1978, respectively.

From 1973 to 1975 , he held a Research Fellowship of the European Space Agency at the Laboratory for Systems Architecture and Analysis (LAAS), Toulouse, France. From 1975 to 1978, he was a Teaching/Research Assistant and subsequently a Research Associate in the Department of Electrical Engineering at the University of Southern California, Los Angeles. In 1979, he was with the Philips Research Laboratory, Brussels, Belgium. In 1980, he returned to the University of Southern California, where he is currently a Professor of Electrical Engineering and a member of the Center for Applied Mathematical Sciences (CAMS). He has also had short-term academic appointments with the Australian National University and the University of Namur, Belgium. His consulting and other activites include the Max Planck Institute, Germany, Memorial Medical Center of Long Beach, CA, Honeywell, Minneapolis, MN, The Aerospace Corporation, El Segundo, CA, Lockheed-Martin, Palmdale, CA, and American GNC Corporation, Simi Valley, CA. His current research interests include modeling complicated nonlinear dynamics, network flow control, and network hyperbolic geometry. 